\title{
Polynuclear Aromatic Hydrocarbons of Tobacco Smoke: Isolation and Identification*
}

\author{
by M. E. Snook, R. F. Severson, H. C. Higman, R. F. Arrendale, and O. T. Chortyk \\ Tobacco Laboratory, Agricultural Research Service, United States Department of Agriculture, \\ Athens, Georgia, U.S.A.
}

\section{INTRODUCTION}

Fractionations of cigarette smoke condensate (CSC) for bioassay have resulted in the isolation of a neutral subfraction possessing high biological activity (1-4). Subfraction F-20 (Fig. 1) has been shown to contain the polynuclear aromatic hydrocarbons (PAH) known to occur in $\operatorname{CSC}(5,6)$. Accordingly, the biological activity of this fraction has been ascribed to the PAH. Therefore, it became important to isolate and identify the PAH in this fraction. More recently, fraction F-20 was further fractionated for bioassay and the PAH successfully concentrated by preparative gel filtration (GF) dromatography (7). The success of the GF procedure stimulated the identification efforts, since, until now, interfering substances have deterred a complete analysis of the PAH in F-20 and similar neutral fractions.
Utilizing our experience with gel filtration chromatography of PAH-containing CSC fractions $(8,9)$, we successfully separated the PAH of F-2o into relatively pure subfractions. Virtually all of the volatile constituents of the subfractions were identified by a combination of gas chromatographic, ultraviolet, and mass spectrometric methods. The methods of identification are detailed and the PAH are tabulated and discussed.

\section{EXPERIMENTAL}

\section{Materials}

All solvents used were Mallindkrodt** "nanograde" or Burdick and Jadkson "distilled-in-glass" grade. Dimethylsulfoxide (DMSO) was Mallinckrodt analytical reagent

Figure 1. Abbrevlated CSC fractionation and PAH lsolation scheme.

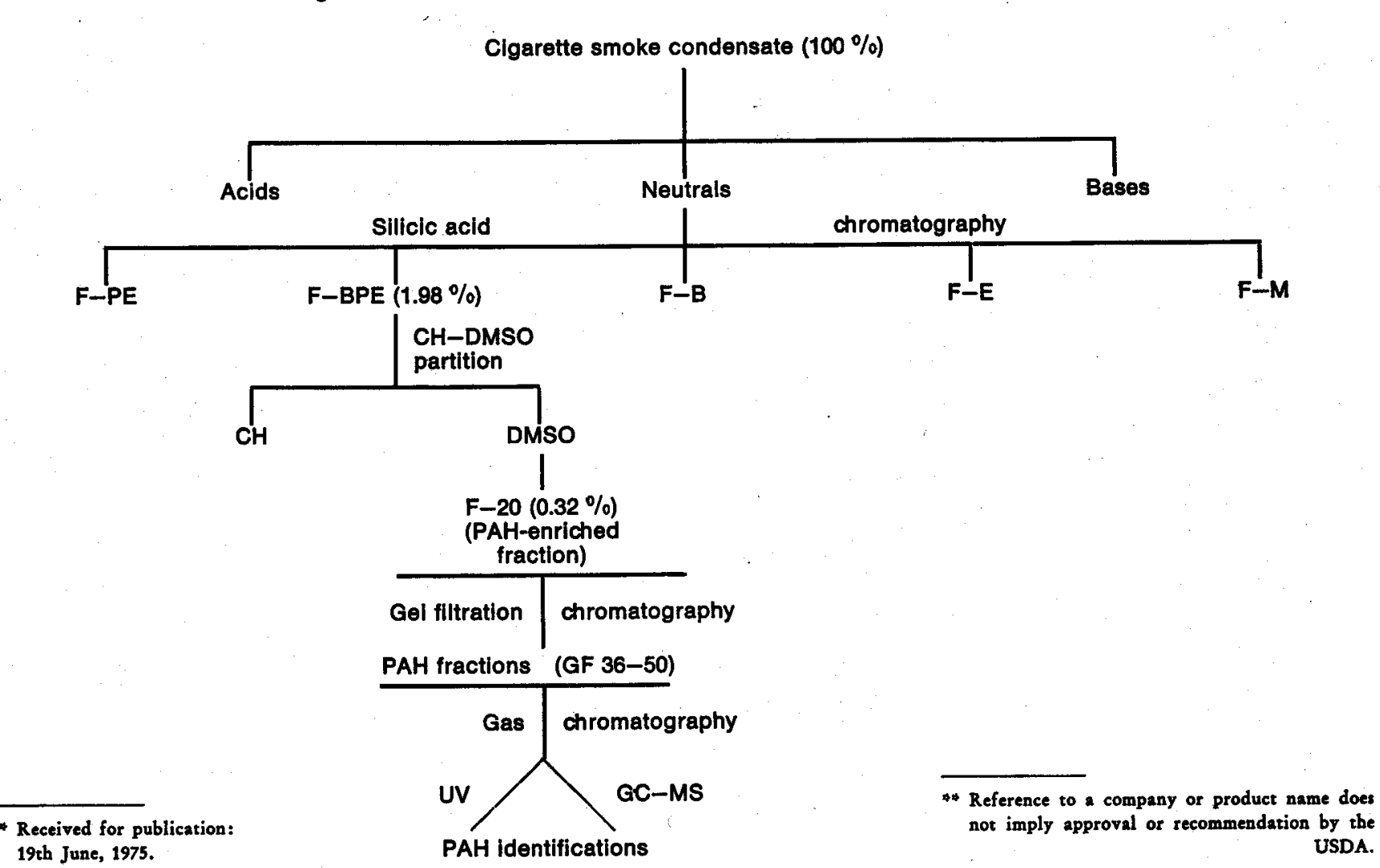


grade. CSC was prepared from commercial, non-filter cigarettes at the Roswell Park Memorial Institute and shipped to us under conditions previously described (xo). The PAH standards were obtained from various commercial sources and used as received. Samples of the six isomeric methyldhrysenes were kindly supplied by Dr. D. Hoffmann, American Health Foundation, Health Researdh Laboratory, Valhalla, New York.

\section{Fractionation of CSC}

The PAH-containing F-20 (Fig. 1) was obtained as described before (2). Acid-base extractions of $1 \mathrm{~kg}$ of CSC yielded a neutral fraction, which was diromatographed on a silicic acid column. Elution with petroleum ether (PE) followed by $25 \%$ benzene $(B)$ in PE yielded fraction F-BPE. Partition of F-BPE between cyclohexane (CH) and DMSO yielded F-2o.

\section{Gel Filtration Chromatography}

The gel filtration (GF) system consisted of four $1.25 \mathrm{~cm} \times 109 \mathrm{~cm}$ Chromatronix LC columns, connected in series and packed with Bio-Beads SX-12 (a neutral, porous, styrene-divinylbenzene copolymer, $M$. exclusion 400, Bio-Rad Laboratories) in benzene. Total length of the wet gel bed was $400 \mathrm{~cm}$ (approximately $200 \mathrm{~g}$ of dry beads). Samples were placed on the gel column with a $1.0 \mathrm{ml}$ injection loop. The eluting solvent was benzene, pumped by a Chromatronix CMP-3 pump at a rate of $120 \mathrm{ml} / \mathrm{h} ; 8 \mathrm{ml}$ fractions were collected. The effluent from the column was monitored at $280 \mathrm{~nm}$ with an Isco Model UA-5 absorbance monitor equipped with a micro-flowcell. The GF system was tested with known PAH and gave reproducible elution volumes for standard PAH, as found before (8). The total sample of F-20 $(3.5 \mathrm{~g})$ was dhromatographed in lots of 0.5 g. Material eluted in GF fractions 24-50. Fractions with the same number were combined for subsequent gas dhromatography (GC). Figure 2 contains the $280 \mathrm{~nm}$ UV trace of the elution of $2 \mathrm{mg}$ of F-20 from the gel columns.

\section{Analytical GC}

GF fractions 40 through 45 were subjected to GC on a Hewlett-Packard Model 5750 gas chromatograph, equipped with $10^{\prime} \times 1 / 8^{\prime \prime}$ and $15^{\prime} \times 1 / 8^{\prime \prime}$ stainless steel (SS) columns, packed with $3 \%$ Dexsil 300 GC on 100/120 mesh Chromosorb W-AW (temperature program, go- $325^{\circ} \mathrm{C}$ at $2^{\circ} / \mathrm{min} ; 48 \mathrm{ml} / \mathrm{min} \mathrm{He}$; injection temperature, $290^{\circ} \mathrm{C}$; flame detector, $350^{\circ} \mathrm{C}$ ). An Autolab System IV Integrator was used to determine peak areas of the GC volatiles. GF fractions 40 and 45 were analyzed on the $15^{\prime}$ column and GF fractions 41-44 were analyzed on the $10^{\prime}$ column.

\section{Preparative GC}

A Hewlett-Padkard Model 5750 gas chromatograph equipped with a thermal conductivity (TC) detector was used for the preparative GC of GF fractions $4^{\circ}$,
41, 43, and 45. The PAH were collected, as indicated below, at the exit port of the TC detector.

\section{Preparative GC of GF Fraction 40}

The compounds in GF fraction 40 were batch collected in six subfractions (into dry ice-cooled vials) from a $15^{\prime} \times 1 / 8^{\prime \prime}$ SS column, padked with $5 \%$ Dexsil on 10o/x20 mesh Chromosorb W-AW, at the same temperature conditions as above. Subfractions were collected according to the following relative retention time (RRT) intervals: GF 40-1, 0.113-0.308 RRT; GF 40-2, 0.324-0.476 RRT; GF 40-3, 0.520-0.610 RRT; GF 40-4, 0.629-0.821 RRT; GF 40-5, 0.842-1.107 RRT; and GF 40-6, 1.124 RRT and above. GF 40-1 was rechromatographed on the $15^{\prime}$ column and the PAH were collected by bubbling the GC effluent into vials containing $95 \%$ EtOH. GC program conditions were: $70^{\circ} \mathrm{C}$ for $10 \mathrm{~min}$, followed by a $2 \%$ min program, until all compounds had eluted. A total of 21 collection cuts were taken. GF $4^{0-2}$ was diromatographed at $120^{\circ} \mathrm{C}$, and 28 collection cuts were trapped in U-shaped capillary tubes, cooled with dry ice. The PAH in GF $40-3$, GF 40-4 and GF 40-5 were collected from the Dexsil column isothermally at $150^{\circ} \mathrm{C}$ (22 collection cuts), at $175^{\circ} \mathrm{C}$ ( 28 collection cuts), and at $175^{\circ} \mathrm{C}$ (43 collection cuts), respectively. GF 40-6 was separated into 53 collection cuts using the following temperature program: $240^{\circ} \mathrm{C}$ for $20 \mathrm{~min}$, followed by an increase of $1 \% \mathrm{~min}$ to $275^{\circ} \mathrm{C}$.

\section{Preparative GC of Fraction 41}

GF fraction 41 was first batch collected into 5 subfractions (GF 4I-1, 0.165-0.350 RRT; GF 41-2, 0.360-0.503 RRT; GF 41-3, 0.520-0.754 RRT; GF 41-4, $0.760-0.877 \mathrm{RRT}$; and GF 41-5, 0.896-1.359 RRT) from a $10^{\prime} \times 3 / 16^{\prime \prime}$ SS $5 \%$ Dexsil column (100/120 mesh Chromosorb W-AW, $100-325^{\circ} \mathrm{C}$ at $2 \% \mathrm{~min}, 35 \mathrm{ml} / \mathrm{min}$ $\mathrm{He}$, injection temperature $290^{\circ} \mathrm{C}$, thermal conductivity detector at $350^{\circ} \mathrm{C}$ ). This column $\left(75-125^{\circ} \mathrm{C}\right.$ at $\left.2^{\circ} \% \mathrm{~min}\right)$ was used to separate the PAH in GF $41-x$ into 16 cuts, which were collected in vials containing $95 \% \mathrm{EtOH}$. GF 41-2 was subjected to preparative GC on the $10^{\prime}$ column $\left(120^{\circ} \mathrm{C} ; 35 \mathrm{ml} / \mathrm{min} \mathrm{He}\right)$ and the PAH were trapped in dry ice-cooled capillary tubes (22 collection cuts). The PAH in GF 41-3, GF 41-4, and GF 41-5 were collected in capillary tubes at room temperature under the following conditions: $150^{\circ} \mathrm{C}, 35 \mathrm{ml} / \mathrm{min} \mathrm{He}$ ( 28 collection cuts); $170^{\circ} \mathrm{C}, 20 \mathrm{ml} / \mathrm{min} \mathrm{He}$ ( $18 \mathrm{col}-$ lection cuts); and $170-325^{\circ} \mathrm{C}$ at $2 \% \mathrm{~min}, 35 \mathrm{ml} / \mathrm{min} \mathrm{He}$ ( 53 collection cuts), respectively.

\section{Preparative GC of GF Fractions 43 and 45}

GF fraction 43 was subjected to preparative GC on a $10^{\prime} \times 1 / 8^{\mu}$ SS $5 \%$ Dexsil column (100-325 $\mathrm{C}$ at $2 \% \mathrm{~min}, 20 \mathrm{ml} / \mathrm{min} \mathrm{He}$ ) and 105 cuts were collected in glass capillary tubes. GF fraction 45 was also separated on this column (150-340 $0^{\circ} \mathrm{C}$ programming at $2 \%$ min, $20 \mathrm{ml} / \mathrm{min} \mathrm{He}$ ) and the PAH were collected in 44 cuts. 


\section{Ultraviolet Spectral Data}

The glass capillary tubes containing the PAH from the above preparative GC runs were rinsed individually into $0.4 \mathrm{ml}$ cuvettes with $95 \%$ ethanol. UV spectra were obtained with a Beckman Acta C III spectrophotometer.

\section{GC-Mass Spectral Data}

A Varian 1400 GC instrument was interfaced with a DuPont 21-492 mass spectrometer. The gas chromatograph was equipped with a 10' $\times 1 / 8^{\prime \prime}$ SS column packed with $5 \%$ Dexsil 300 GC on 100/120 Chromosorb W-AW (injection temperature, $290^{\circ} \mathrm{C}$; FID, $350^{\circ} \mathrm{C}$; and $20 \mathrm{ml} / \mathrm{min} \mathrm{He}$ ). GF subfractions $40-1,-2$, $-3,-4,-5$, and -6 were chromatographed isothermally at $100,120,165,185,240$ and $285^{\circ} \mathrm{C}$, respectively. GF fractions 41,43 , and 45 were chromatographed using a temperature program of $2 \%$ min from $100^{\circ} \mathrm{C}$ to $325^{\circ} \mathrm{C}$.

Mass spectral (MS) analyses were performed as follows. The GC effluent was split $1: 1$, one half going to the FID of the gas chromatograph and the other half to the source area of the mass spectrometer. A jet separator, at $300^{\circ} \mathrm{C}$, stripped helium from the effluent before mass spectral analysis. Mass spectra of effluent GC peaks were obtained under the following conditions: a scan rate of $100 \mathrm{~s} / \mathrm{mass}$-decade, a minimal resolution of 1000 , and $70 \mathrm{eV}$ electron bombardment. Mass spectra were taken as often as possible during the elution time of a GC peak to determine mass integrity. The spectra were recorded by a high-speed recording oscillograph or an AEI DS-30 computerized data system. Mass spectral data were analyzed by both manual and computer aided techniques.

\section{RESULTS}

The characteristics of the GF step are presented in Fig. 2; the bar graph represents the weight percent of F-20 in each GF fraction. The smoke PAH, like the
PAH standards, began to elute in GF fraction 36. Analytical GC data, such as relative retention times and percent composition of GC volatiles were calculated for GF fractions 40 through 45, inclusive. Chromatograms of GF fractions $40,41,43$, and 45 are presented in Fig. 3, 4, 5, and 6. Peaks having the same RRT have been given the same number in all tables, chromatograms, and figures. This allows the comparison of the individual peak changes in increasing GF fractions. At least 115 peaks are discernable on the dhromatograms. GF fractions 40 and 41 contain many early eluting components not found in GF fractions 43 and 45. Comparison of the chromatograms of GF fractions 40 to 45 shows that [1] many peaks decrease and disappear, [2] others, first increase then decrease, and [3] others mainly increase. This phenomenon will be shown later to be a characteristic of the GF step and actually aided in the identification of minor PAH components and isomeric PAH. Standard PAH were cochromatographed with the GF fractions to determine GC retention time correlation.

Preparative GC was performed on GF fractions 40, 41, 43, and 45. Whenever possible, samples were collected while on the upslope, top, and downslope of the GC peaks to give at least three cuts for each peak. The object of taking multiple cuts of a single peak was to give selective enrichment of components for identification by UV spectroscopy. Analytical GC indicated that GF fractions 42 and 44 contained material also found in the adjacent GF fractions. Therefore, preparative GC separations and analyses on these fractions were not deemed necessary.

The results of the identification and quantitation of the components in the GF fractions are given in Table 1. Peak numbers refer to GC peaks in Figures 3 to 6. Peaks are tabulated in order of their RRT, with peak \#70 (pyrene) equalling 1.000. The percent composition of the GF fraction, based on total GC volatiles and assuming unitary detector response, is also given. The tabulated values for percent composition of the GC peak components in each gel fraction depended on its percentage of total GC volatiles. The percentage

Figure 2. Gel filtration chromatography [A: percent weight distribution of F-20 gel fractions; $B$ : $280 \mathrm{~nm}$ absorbance curve for the elution of F-20 (100 = 1.28 absorbance units); C: elution curves for standard PAH (1 - 3,6-dimethylphenanthrene; 2 - phenanthrene; 3 - pyrene; 4 - benzo(a)pyrene)].

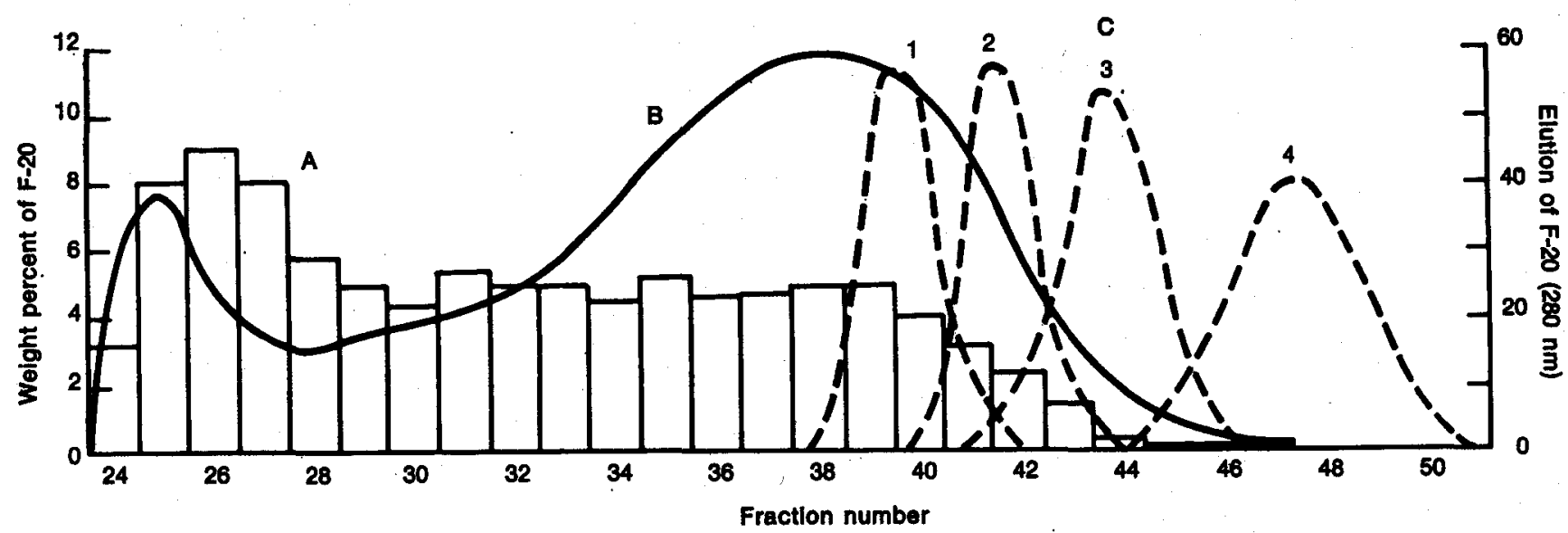




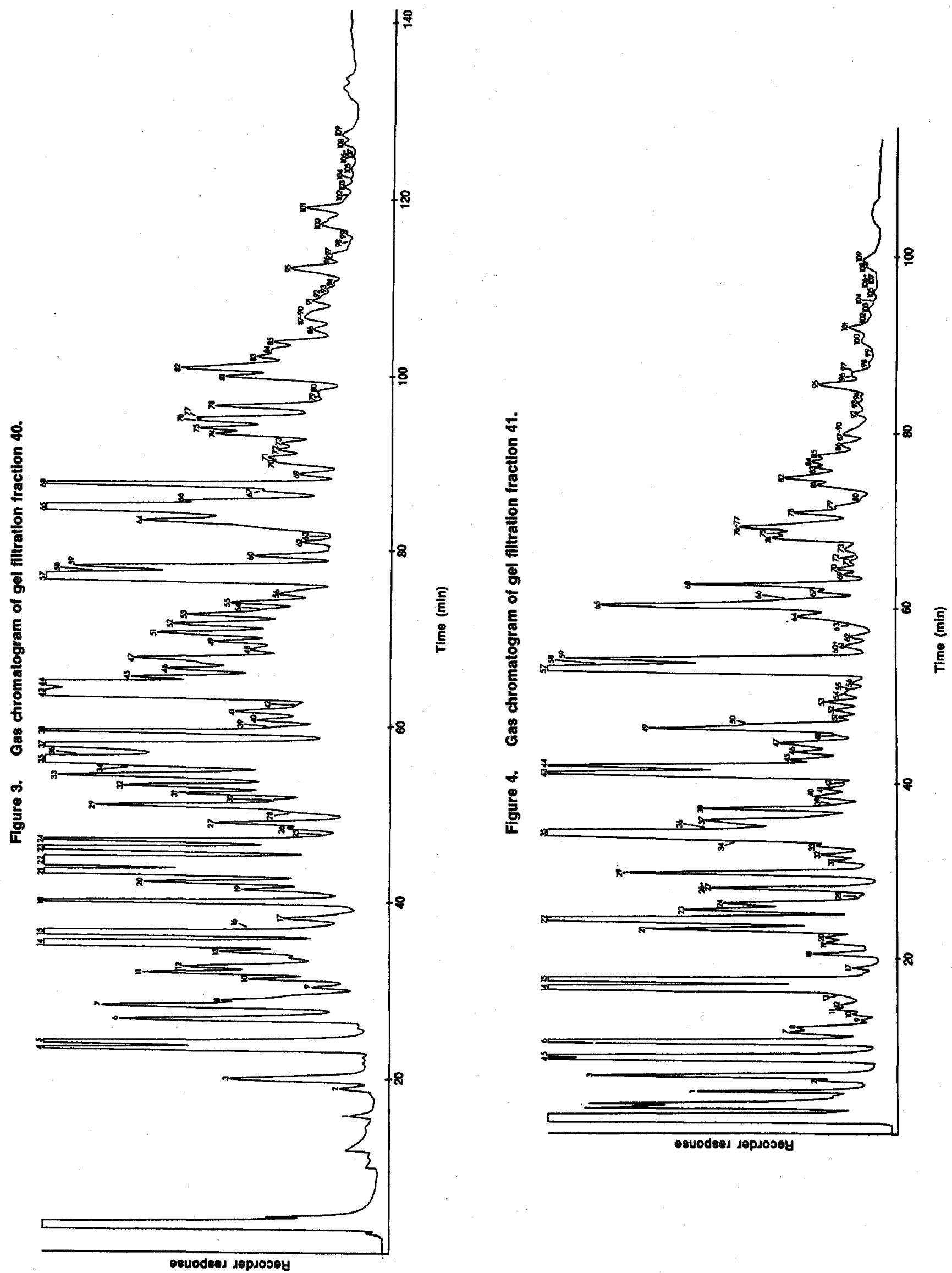



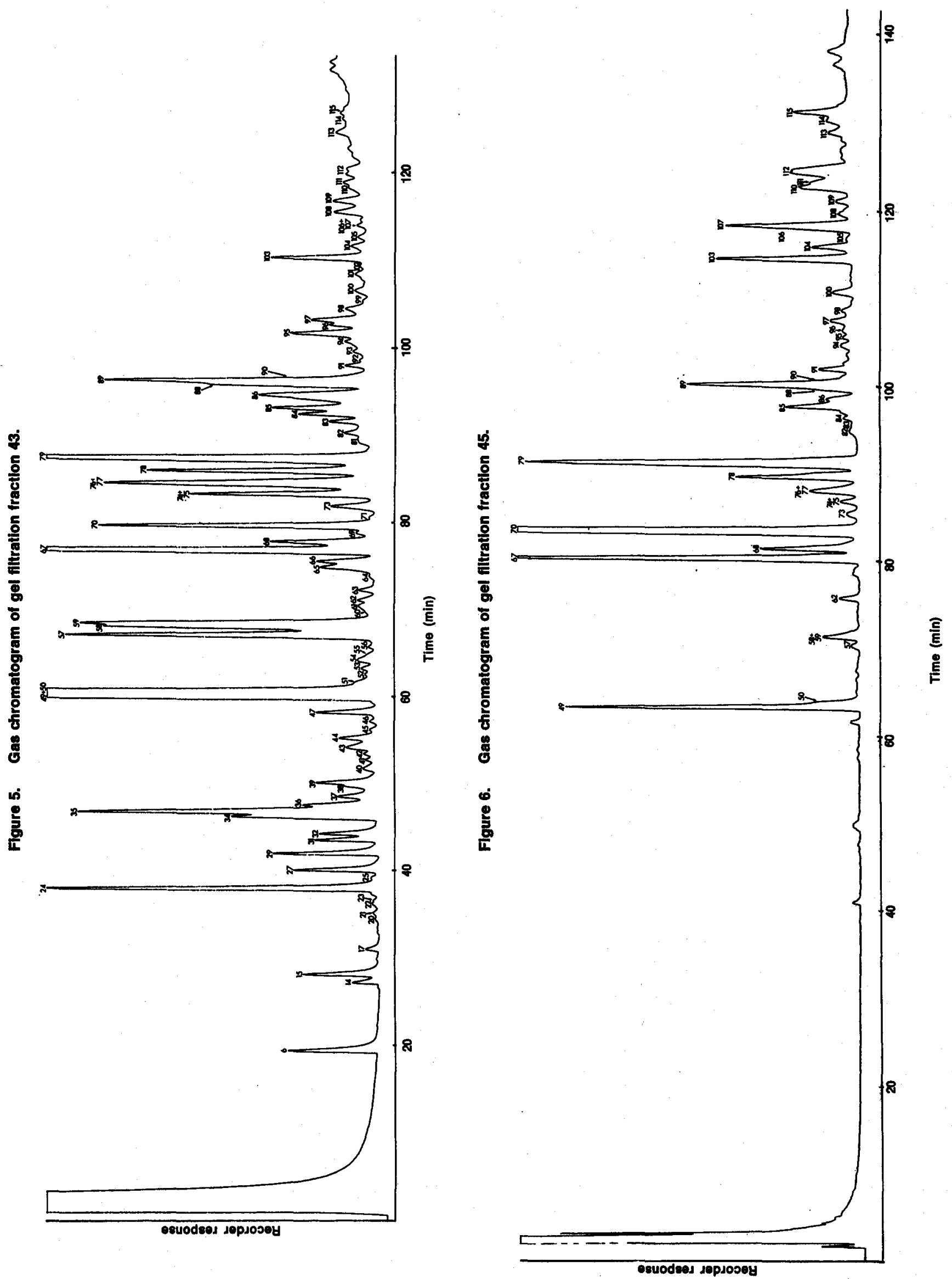
values are strongly representative of increasing or decreasing concentrations of the components in successive gel fractions. Where possible, the major component of a peak is indicated. Frequently, the major component of the same peak changes, in progressing from GF fractions 40 to 45 .

The criteria for identification of components are also indicated in Table 1. When GC retention times and/or literature UV data were unavailable, the data in Table 2 were the basis for identification.

\section{DISCUSSION}

Although numerous analytical methods for the PAH of tobacco smoke have been proposed (11-14), these techniques have utilized relatively crude CSC samples that contained many other compounds in addition to the PAH. By applying analytical GF dromatography to a PAH-enriched fraction ( $\mathrm{F}-2 \mathrm{O})$, we obtained a highly refined PAH-isolate (GF fractions 36 to 50), which represented only $0.01 \%$ of the starting CSC. This GF method was an extension of preparative GF chromatography used to separate F-zo into subfractions for bioassay tests (7). The key to the successful identifications of the PAH was the analysis of each refined GF fraction. The only other compounds, in addition to the PAH that were detected in the GF fractions, were several oxygen-PAH analogues, sudh as benzo(b)furan, naphthofurans, dibenzofuran, and their methylated derivatives. However, this was not unexpected as their properties are very similar to those of PAH. The Bio-Beads SX-12 gels separated PAH from other materials by an adsorption type mechanism, whereby the PAH were retained due to their aromatic character. Another separation factor involved early elution of methylated PAH before the parent PAH compound. Thus, increasing the number of methyl groups on a PAH decreases its elution volume. On the other hand, increasing the number of aromatic rings in a PAH increases its elution volume. These two gel properties have been discussed in detail (9). Fig. 2 shows that the order of elution of the standards was 3,6-dimethylphenanthrene, phenanthrene, pyrene, and benzo(a)pyrene.

The partial separations of PAH by methyl substitution and ring number are also well illustrated by the data for the PAH of CSC. These two factors significantly aided in the identification of the PAH. That is, the methylated small ring PAH were found in the early $G F$ fractions. Subsequent fractions contained the parent compounds and the methylated derivatives of higher ring $\mathrm{PAH}$, while still later fractions contained predominantly the parent PAH. Thus, GF fractions $4^{\circ}$ and 41 were rich in trimethyl- and dimethyl-derivatives of naphthalene, fluorene, phenanthrene, anthracene, fluoranthene, pyrene, and chrysene. GF fractions 40 and 41 were also rich in methyl-derivatives of naphthalene, fluorene, phenanthrene, and anthracene. GF fraction 43 contained large amounts of the methyl-derivatives of phenanthrene, fluoranthene, pyrene, 1,2-benzanthracene, chrysene, benzofluoranthene, benzo(a)pyrene, and benzo(e)pyrene. The parent PAH - phenanthrene, anthracene, benzofluorenes, fluoranthene, pyrene, 1,2 -benzanthracene, chrysene, benzofluoranthenes, acenaphthylene, and benz(f)indene - were concentrated in GF fraction 43. GF fraction 45 contained mainly the parent PAH - phenanthrene, anthracene, fluoranthene, pyrene, benzo $(g, h, i) f$ fluoranthene, chrysene, benzofluoranthene, benzo(a)pyrene, and benzo(e)pyrene. It also contained methyl-derivatives of fluoranthene, pyrene, benzo(a)pyrene, and benzo(e)pyrene.

As mentioned before, the percent composition of gel fractions given in Table $I$ only indicates trends, since the percentages for each peak depended upon the amount and type of GC volatiles. To quantitate individual PAH, calculations must be based on the combined data of all the gel fractions which contain the particular $\mathrm{PAH}$. Such calculations have been performed in conjunction with a rapid analytical method for PAH of cigarette smoke (15).

The components of the GF fractions were identified by a combination of GC, UV, and GC-MS methods. These criteria together with the separation of PAH by GF, according to structure, have made many of the identifications more definitive. In only four cases was identification based on MS data alone. The lack of suitable PAH standards, particularly methyl- and dimethylderivatives, presented some problems in the unambiguous identification of the PAH. In some cases, additional criteria, such as NMR, spectrophotofluorometry, and highspeed liquid dhromatography, will be needed to determine the position of methyls on the ring systems. Work will be continued in these areas. Obviously, previous identifications of PAH based on only one criterion (i.e., UV, MS data, or GC retention time) must be used cautiously.

Currently, work is continuing on the identification of the PAH constituents in GF fractions higher than 45 . The material in these fractions constitutes only a small percentage of the total PAH, but should contain the higher ring $\mathrm{PAH}$ systems, from benzo(a)pyrenes and dibenzopyrenes to coronene and above. Due to the biological activity ascribed to some of the higher molecular weight PAH, this portion of the PAH spectrum should prove to be as interesting and as important as the lower molecular weight PAH.

\section{SUMMARY}

A neutral fraction of cigarette smoke condensate, which had shown biological activity and was known to contain polynuclear aromatic hydrocarbons (PAH), was fractionated by analytical gel filtration chromatography. These gel fractions were subjected to gas chromatographic separation and their components were identified by relative GC retention times, UV spectra, and mass spectral data. More than $300 \mathrm{PAH}$, ranging from indene to the dimethylbenzopyrenes, were characterized. This 
method of isolation has yielded fractions which were more amenable to definitive identifications. The criteria used for identification are tabulated for all the identified PAH compounds.

\section{ZUSAMMENFASSUNG}

Eine neutrale Fraktion des Kondensates von Cigarettenrauch, bei der biologische Aktivität und ein Gehalt an polycyclischen aromatischen Kohlenwasserstoffen (PAH) beobachtet worden waren, wurde mittels analytischer Gel-Chromatographie fraktioniert. Nach gaschromatographischer Trennung dieser Gel-Fraktionen wurden deren Bestandteile auf Grund relativer GC-Retentionszeiten, UV-Spektren und massenspektrometrischen Werten identifiziert. Mehr als $\mathbf{3 0 0}$ polycyclische aromatische Kohlenwasserstoffe - vom Inden bis zum Dimethylbenzpyren - wurden nachgewiesen. Das Trennungsverfahren ergab Fraktionen, die für endgültige Identifizierungen geeigneter waren. Die zum Nachweis benutzten Kriterien werden für alle identifizierten Kohlenwasserstoffverbindungen tabellarisch aufgeführt.

\section{RESUME}

On a fractionné par chromatographie analytique à perméation de gel, une fraction neutre de condensat de fumée de cigarette, dont on a démontré une activité biologique et dont on sait qu'elle contient des hydrocarbures polynucléaires aromatiques (PAH). Ces fractions de "gel" sont soumises à une séparation par dhromatographie en phase gazeuse, et on a identifié les composants par leurs temps de rétention relatifs, leurs spectres UV, et leurs données en spectrographie de masse. On a ainsi démontré la présence de plus de 300 PAH, variant de l'indène aux diméthylbenzopyrènes. Cette méthode d'isolation produit un groupe de fractions qu'il est plus aisé d'identifier avec certitude. Les critères employés pour l'identification de tous lesdits composés PAH sont présentés sous forme de tableau.

\section{REFERENCES}

1. Swain, A. P., J. E. Cooper, R. L. Stedman, and F. G. Bock: Beitr. Tabakforsch. 5 (1969) 109.

2. Bock, F. G., A. P. Swain, and R. L. Stedman: J. Natl. Cancer Inst. 44 (1970) 1305.

3. Bock, F. G., A. P. Swain, and R. L. Stedman: J. Natl. Cancer Inst. 49 (1972) 477.

4. Swain, A. P., F. G. Bodk, J. E. Cooper, W. J. Chamberlain, E. D. Strange, L. Lakritz, and R. L. Stedman: Beitr. Tabakforsch. 7 (1973) 1.

5. Stedman, R. L., R. L. Miller, L, Lakritz, and W. J. Chamberlain: Chem. and Ind. 1968, 394.

6. Miller, R. L., W. J. Chamberlain, and R. L. Stedman: Tob. Sci. 13 (xg69) 21.

7. Chamberlain, W. J., D. B. Walters, M. E. Snook, O. T. Chortyk, and F. J. Akin: Beitr. Tabakforsch. 8 (1975) 133.

8. Snook, M. E., W. J. Chamberlain, R. F. Severson, and O. T. Chortyk: Anal. Chem. 47 (1975) 2155.

9. Snook, M. E.: Anal. Chim. Acta. 81 (1976) 423.

10. Swain, A. P., J. E. Cooper, and R. L. Stedman: Cancer Res. 29 ( 1969$) 579$.

11. Carugno, N., and S. Rossi: Anal. Chem. 39 (1967) 103.

12. Davis, H. J.: Talanta 16 (1969) 621.

13. Rathkamp, G., and D. Hoffmann: Information Bulletin CORESTA 1972-Special, p. 16.

14. Hoffmann, D., G. Rathkamp, K. D. Brunnemann, and E. L. Wynder: Science of the Total Environment 2 (1973) 157.

15. Severson, R. F., M. E. Snook, O. T. Chortyk, and R. F. Arrendale: Beitr. Tabakforsh. 8 (1976) 273.

16. UV atlas of organic compounds; Plenum Press, New York, 1966.

17. Entwistle, I. D., and R. A. W. Johnstone: J. Chem. Soc. (C) 1968, 1818.

18. Stubbs, H. W. D., and S. H. Tucker: J. Chem. Soc. 1954, 227.

19. Clar, E.: Aromatische Kohlenwasserstoffe, polycyclische Systeme; Springer-Verlag, Berlin - Göttingen - Heidelberg, 1952.

The authors' address:

Tobacco Laboratory, Agricultural Research Seroice, U.S. Dept. of Agriculture, Athens, Georgia, 30604, U.S.A. 
Table 1. Composition of gel filtration fractions 40 to 45 .

\begin{tabular}{|c|c|c|c|c|c|c|c|c|c|c|c|}
\hline \multirow{2}{*}{$\begin{array}{l}\text { Peak } \\
\text { No. }\end{array}$} & \multirow{2}{*}{ Compound } & \multirow{2}{*}{$\begin{array}{c}\text { Relative } \\
\text { reten- } \\
\text { tion } \\
\text { timea }\end{array}$} & \multicolumn{6}{|c|}{ Gel fraction } & \multicolumn{3}{|c|}{$\begin{array}{l}\text { Criteria of } \\
\text { identification }\end{array}$} \\
\hline & & & \multicolumn{6}{|c|}{ Percent compositionb } & GC-RTC & UVd & MSe \\
\hline 1 & Indene & 0.113 & 0.06 & 1.27 & 0.04 & $<0.01$ & - & - & + & + & + \\
\hline 2 & Methylbenzo(b)furan & 0.156 & 0.11 & 0.53 & 0.02 & $<0.01$ & - & - & & + & + \\
\hline 3 & Methylbenzo(b)furan & 0.165 & 0.66 & 2.52 & 0.15 & $<0.01$ & - & - & & + & + \\
\hline 4 & $\begin{array}{l}\text { 1-Methylindene } \\
\text { 3-Methylindene }\end{array}$ & 0.198 & $\begin{array}{r}1.09 \\
M^{f}\end{array}$ & $\begin{array}{r}1.58 \\
M\end{array}$ & 0.10 & $<0.01$ & - & - & + & $+(16)$ & + \\
\hline 5 & Methylindene & 0.206 & 1.86 & 2.31 & 0.12 & $<0.01$ & - & - & & + & + \\
\hline 6 & $\begin{array}{l}\text { Naphthalene } \\
\text { Dimethylbenzo(b)furan }\end{array}$ & 0.238 & $\begin{array}{r}1.08 \\
M\end{array}$ & $\begin{array}{r}3.55 \\
M\end{array}$ & 6.94 & 1.13 & $<0.01$ & - & + & + & + \\
\hline 7 & Dimethylbenzo(b)furans & 0.256 & 1.78 & 0.89 & 0.07 & $<0.01$ & - & - & & + & + \\
\hline 8 & Dimethylindene & 0.259 & & 0.769 & & $<0.01$ & - & - & & + & + \\
\hline 9 & $\begin{array}{l}\text { Dimethylbenzo(b)furans } \\
\text { Dimethylindene }\end{array}$ & 0.285 & $\begin{array}{r}0.19 \\
M\end{array}$ & $\begin{array}{r}0.20 \\
M\end{array}$ & $<0.01$ & - & - & - & & $\stackrel{+}{+}$ & $\begin{array}{l}+ \\
+\end{array}$ \\
\hline 10 & Dimethylindene & 0.292 & 0.37 & 0.24 & $<0.01$ & - & - & - & & + & + \\
\hline 11 & Dimethylindene & 0.300 & 0.78 & 0.36 & $<0.01$ & - & - & - & & + & + \\
\hline 12 & Dimethylindene & 0.308 & 0.97 & 0.55 & $<0.01$ & - & - & - & & + & + \\
\hline 13 & Dimethylindene & 0.327 & 0.76 & & 0.05 & $<0.01$ & - & - & & + & + \\
\hline 14 & 2-Methylnaphthalene & 0.335 & 5.79 & 6.99 & 2.00 & 0.28 & $<0.01$ & - & + & + & + \\
\hline 15 & 1-Methylnaphthalene & 0.350 & 4.64 & 6.46 & 3.87 & 0.98 & $<0.01$ & - & + & + & + \\
\hline 16 & Trimethylindene & & & & & & & - & & + & + \\
\hline 17 & Trimethylindenes & 0.372 & 0.41 & 0.43 & $<0.01$ & - & - & - & & + & + \\
\hline 18 & $\begin{array}{l}\text { Biphenyl } \\
\text { Trimethylindenes } \\
\text { Trimethylbenzo(b)furan }\end{array}$ & 0.396 & $\begin{array}{r}1.29 \\
M\end{array}$ & $\begin{array}{r}0.54 \\
M\end{array}$ & $<0.01$ & - & - & - & + & $\begin{array}{l}+ \\
+ \\
+\end{array}$ & $\begin{array}{l}+ \\
+ \\
+\end{array}$ \\
\hline 19 & $\begin{array}{l}\text { Trimethylindene } \\
\text { Trimethylbenzo(b)furan }\end{array}$ & 0.412 & 0.57 & 0.56 & 0.16 & 0.02 & $<0.01$ & - & & + & $\begin{array}{l}+ \\
+\end{array}$ \\
\hline 20 & $\begin{array}{l}\text { 2-Ethylnaphthalene } \\
\text { 1-Ethylnaphthalene }\end{array}$ & 0.422 & 0.88 & 0.47 & 0.16 & 0.04 & $<0.01$ & - & $\begin{array}{l}+ \\
+\end{array}$ & $\begin{array}{l}+ \\
+\end{array}$ & $\begin{array}{l}+ \\
+\end{array}$ \\
\hline
\end{tabular}


Table 1. Composition of gel filtration fractions 40 to 45 (contd.).

\begin{tabular}{|c|c|c|c|c|c|c|c|c|c|c|c|}
\hline \multirow{2}{*}{$\begin{array}{c}\text { Peak } \\
\text { No. }\end{array}$} & \multirow{2}{*}{ Compound } & \multirow{2}{*}{$\begin{array}{c}\text { Relative } \\
\text { reten- } \\
\text { tion } \\
\text { timea }\end{array}$} & \multicolumn{6}{|c|}{ Gel fraction } & \multicolumn{3}{|c|}{$\begin{array}{c}\text { Criteria of } \\
\text { Identification }\end{array}$} \\
\hline & & & \multicolumn{6}{|c|}{ Percent compositionb } & GC-RTC & UVd & MSe. \\
\hline 21 & $\begin{array}{l}\text { 2,6-Dimethylnaphthalene } \\
\text { 2,7-Dimethylnaphthalene } \\
\text { 1-Vinylnaphthalene }\end{array}$ & 0.434 & $\begin{array}{r}2.96 \\
M\end{array}$ & $\begin{array}{r}1.56 \\
M\end{array}$ & 0.30 & 0.07 & $<0.01$ & - & $\begin{array}{l}+ \\
+ \\
+\end{array}$ & $\begin{array}{l}+ \\
+(16)\end{array}$ & $\begin{array}{l}+ \\
+ \\
+\end{array}$ \\
\hline 22 & $\begin{array}{l}\text { 1,3-Dimethylnaphtalene } \\
\text { 1,6-Dimethylnaphthalene } \\
\text { 2-Vinylnaphthalene }\end{array}$ & 0.450 & 7.67 & 4.21 & 0.63 & 0.09 & $<0.01$ & - & $\begin{array}{l}+ \\
+ \\
+\end{array}$ & $\begin{array}{l}+ \\
+ \\
+\end{array}$ & $\begin{array}{l}+ \\
+ \\
+\end{array}$ \\
\hline 23 & $\begin{array}{l}\text { 2,3-Dimethylnaphthalene } \\
\text { 1,4-Dimethylnaphthalene } \\
\text { 1,5-Dimethylnaphthalene }\end{array}$ & 0.467 & 2.16 & 1.40 & 3.72 & 0.12 & $<0.01$ & - & $\begin{array}{l}+ \\
+ \\
+\end{array}$ & $\begin{array}{l}+ \\
+ \\
+\end{array}$ & $\begin{array}{l}+ \\
+ \\
+\end{array}$ \\
\hline 24 & $\begin{array}{l}\text { 1,7-Dimethylnaphthalene } \\
\text { Acenaphthylene }\end{array}$ & 0.476 & $\begin{array}{r}1.33 \\
M\end{array}$ & $\begin{array}{r}1.46 \\
\cdot M\end{array}$ & & $\begin{array}{r}3.52 \\
M\end{array}$ & 1.25 & $\begin{array}{l}0.17 \\
M\end{array}$ & + & $\begin{array}{l}+(17) \\
+\end{array}$ & $\begin{array}{l}+ \\
+\end{array}$ \\
\hline 25 & 3-Methylbiphenyl & 0.489 & $0.15^{h}$ & 0.10 & 0.11 & 0.07 & $<0.01$ & - & + & + & + \\
\hline 26 & 4-Methylbiphenyl & 0.497 & $0.26 h$ & & $<0.01$ & - & - & - & + & + & + \\
\hline 27 & $\begin{array}{l}\text { 1,8-Dimethylnaphthalene } \\
\text { Acenaphthene } \\
\text { Trimethylnaphthalene }\end{array}$ & 0.503 & 0.64 & 1.32 & 1.55 & 0.90 & 0.13 & $<0.01$ & + & $\stackrel{+}{+}$ & $\begin{array}{l}+ \\
+ \\
+\end{array}$ \\
\hline 28 & Trimethylnaphthalenes & 0.526 & 1.32 & 1.89 & 2.09 & 1.14 & 0.21 & $<0.01$ & & + & + \\
\hline 29 & Dibenzofuran & & $\mathbf{M}$ & $\mathbf{M}$ & & $\mathbf{M}$ & & & + & + & + \\
\hline 30 & Trimethylnaphthalene & 0.537 & 0.31 & . & $<0.01$ & - & - & - & & + & + \\
\hline 31 & $\begin{array}{l}\text { Trimethylnaphthalene } \\
\text { Unknown }\end{array}$ & 0.545 & $\begin{array}{r}0.74 \\
M\end{array}$ & 0.38 & 0.66 & $\begin{array}{r}0.63 \\
M\end{array}$ & 0.26 & $\begin{array}{r}0.07 \\
M\end{array}$ & & $\begin{array}{l}+ \\
+\end{array}$ & $\stackrel{+}{+}$ \\
\hline 32 & $\begin{array}{l}\text { Trimethylnaphthalene } \\
\text { Naphtho( } 2,1-b) \text { furan }\end{array}$ & 0.555 & $\begin{array}{r}1.06 \\
M\end{array}$ & $\begin{array}{r}0.52 \\
M\end{array}$ & 0.53 & $\begin{array}{c}0.72 \\
M\end{array}$ & 0.35 & $\begin{array}{l}0.09 \\
M\end{array}$ & & $\begin{array}{l}+ \\
+(18)\end{array}$ & $\begin{array}{l}+ \\
+\end{array}$ \\
\hline 33 & Trimethylnaphthalene & 0.568 & $1.80 \mathrm{~h}$ & $0.55^{h}$ & $<0.01$ & - & - & - & & + & + \\
\hline 34 & $\begin{array}{l}\text { Trimethylnaphthalene } \\
\text { 1-Methylacenaphthylene }\end{array}$ & 0.581 & $\stackrel{0.86 h}{M}$ & $h$ & . & $\begin{array}{c}1.51 \mathrm{~h} \\
\mathrm{M}\end{array}$ & 1.76 & $<0.01$ & . & $\stackrel{+}{+(19)}$ & $\begin{array}{l}+ \\
+\end{array}$ \\
\hline 35 & $\begin{array}{l}\text { Fluorene } \\
\text { Methylacenaphthylene } \\
\text { Trimethylnaphthalene }\end{array}$ & 0.588 & $\begin{array}{r}5.40 \\
M\end{array}$ & $\begin{array}{r}8.50 \\
M\end{array}$ & 10.13 & $\begin{array}{r}3.76 \\
M\end{array}$ & & 0.33 & + & $\begin{array}{l}+ \\
+ \\
+\end{array}$ & $\begin{array}{l}+ \\
+ \\
+\end{array}$ \\
\hline 36 & $\begin{array}{l}\text { 9-Methylfluorene } \\
\text { Trimethylnaphthalene } \\
\text { Methylacenaphthylenes }\end{array}$ & 0.596 & & & & $\begin{array}{r}0.84 \\
M\end{array}$ & : & $<0.01$ & + & $\begin{array}{l}+ \\
+ \\
+\end{array}$ & $\begin{array}{l}+ \\
+ \\
+\end{array}$ \\
\hline
\end{tabular}




\begin{tabular}{|c|c|c|c|c|c|c|c|c|c|c|c|}
\hline \multirow{2}{*}{$\begin{array}{l}\text { Peak } \\
\text { No. }\end{array}$} & \multirow{2}{*}{ Compound } & \multirow{2}{*}{$\begin{array}{c}\text { Relative } \\
\text { reten- } \\
\text { tion } \\
\text { timea }\end{array}$} & \multicolumn{6}{|c|}{ Gel fraction } & \multicolumn{3}{|c|}{$\begin{array}{c}\text { Criteria of } \\
\text { identification }\end{array}$} \\
\hline & & & \multicolumn{6}{|c|}{ Percent compositionb } & GC-RTC & UVd & MS* \\
\hline 37 & $\begin{array}{l}\text { Methylacenaphthene } \\
\text { Trimethylnaphthalene } \\
\text { Unknown }\end{array}$ & 0.612 & $\begin{array}{r}2.69 \\
M\end{array}$ & $\begin{array}{r}2.34 \\
M\end{array}$ & 1.48 & $\begin{array}{r}0.61 \\
M\end{array}$ & $<0.01$ & - & & $\begin{array}{l}+ \\
+\end{array}$ & $\begin{array}{l}+ \\
+ \\
+\end{array}$ \\
\hline 38 & $\begin{array}{l}\text { Methyldibenzofuran } \\
\text { Unknown }\end{array}$ & 0.629 & $\begin{array}{r}1.68 \\
M\end{array}$ & 1.38 & 1.00 & $\begin{array}{r}0.36 \\
M\end{array}$ & $<0.01$ & - & & $\stackrel{+}{+}$ & + \\
\hline 39 & Benz(f)indene & 0.636 & & & & 0.80 & 0.20 & $<0.01$ & & $+(18)$ & + \\
\hline 40 & $\begin{array}{l}\text { Methyl-unknown of RRT } 0.545 \\
\text { Dimethylacenaphthene }\end{array}$ & 0.647 & $\begin{array}{r}0.58 \\
M\end{array}$ & $\begin{array}{r}0.75 \\
M\end{array}$ & 0.40 & 0.29 & $<0.01$ & - & & + & + \\
\hline 41 & $\begin{array}{l}\text { Dimethylacenaphthene } \\
\text { Methyl-unknown of RRT } 0.545\end{array}$ & 0.660 & $\begin{array}{r}0.92 \\
M\end{array}$ & 0.57 & 0.16 & $\begin{array}{r}0.14 \\
M\end{array}$ & $<0.01$ & - & & $\begin{array}{l}+ \\
+\end{array}$ & + \\
\hline 42 & Dimethylacenaphthene & 0.671 & h & $0.23 \mathrm{~h}$ & 0.23 & $0.16 \mathrm{~h}$ & $<0.01$ & - & & + & + \\
\hline 43 & $\begin{array}{l}\text { 2-Methylfluorene } \\
\text { 3-Methylfluorene } \\
\text { Dimethylacenaphthylene } \\
\text { Methyl-unknown of RRT } 0.545\end{array}$ & 0.682 & $\begin{array}{r}5.10 \\
M\end{array}$ & $\begin{array}{r}3.11 \\
M\end{array}$ & 1.00 & M & 0.09 & 0.10 & $\begin{array}{l}+ \\
+(13)\end{array}$ & $\begin{array}{l}+ \\
+ \\
+\end{array}$ & $\begin{array}{l}+ \\
+ \\
+ \\
+\end{array}$ \\
\hline 44 & $\begin{array}{l}\text { 1-Methylfluorene } \\
\text { 4-Methylfluorene } \\
\text { Dimethylacenaphthylene } \\
\text { Methyldibenzofuran }\end{array}$ & 0.694 & $\begin{array}{r}3.92 \\
M \\
M\end{array}$ & $\begin{array}{r}2.44 \\
M \\
M\end{array}$ & 0.90 & $\begin{array}{r}0.53 \\
M\end{array}$ & 0.16 & 0.09 & $\begin{array}{l}+ \\
+(13)\end{array}$ & $\begin{array}{l}+ \\
+(16) \\
+ \\
+\end{array}$ & $\begin{array}{l}+ \\
+ \\
+ \\
+\end{array}$ \\
\hline 45 & $\begin{array}{l}\text { Dimethylacenaphthene } \\
\text { Dimethylacenaphthylenes } \\
\text { Dimethyldibenzofuran }\end{array}$ & 0.704 & $\begin{array}{r}1.03 \\
M\end{array}$ & $\begin{array}{r}0.76 \\
M \\
M\end{array}$ & 0.14 & 0.14 & $<0.01$ & - & & $\begin{array}{l}+ \\
+ \\
+\end{array}$ & $\begin{array}{l}+ \\
+ \\
+\end{array}$ \\
\hline 46 & $\begin{array}{l}\text { Dimethylacenaphthene } \\
\text { Dimethyldibenzofuran } \\
\text { Methylbenz(f)indene }\end{array}$ & 0.716 & $\begin{array}{r}0.91 \\
M\end{array}$ & 0.69 & 0.02 & 0.12 & $<0.01$ & - & & $\begin{array}{l}+ \\
+ \\
+\end{array}$ & $\begin{array}{l}+ \\
+ \\
+\end{array}$ \\
\hline 47 & $\begin{array}{l}\text { Dimethylacenaphthene } \\
\text { Dimethyldibenzofuran } \\
\text { Methylbenz(f)indene } \\
\text { Tetramethylnaphthalene }\end{array}$ & 0.731 & 1.69 & 1.21 & 0.44 & 0.709 & 0.51 & 0.23 & & $\begin{array}{l}+ \\
+ \\
+ \\
+\end{array}$ & $\begin{array}{l}+ \\
+ \\
+ \\
+\end{array}$ \\
\hline 48 & $\begin{array}{l}\text { Trimethylacenaphthene } \\
\text { Trimethylacenaphthylene } \\
\text { Methylbenz(f)indene } \\
\text { Dimethyl-unknown of RRT } 0.545\end{array}$ & 0.745 & 0.41 & & $<0.01$ & - & - & - & & $\begin{array}{l}+ \\
+ \\
+ \\
+\end{array}$ & $\begin{array}{l}+ \\
+ \\
+ \\
+\end{array}$ \\
\hline 49 & $\begin{array}{l}\text { Phenanthrene } \\
\text { Trimethylacenaphthene }\end{array}$ & 0.754 & $\begin{array}{r}0.76 \\
M\end{array}$ & $\begin{array}{r}2.60 \\
M\end{array}$ & 17.26 & $\begin{array}{r}20.32 \\
M\end{array}$ & 20.87 & $\begin{array}{c}6.75 \\
M\end{array}$ & + & + & $\begin{array}{l}+ \\
+\end{array}$ \\
\hline 50 & Anthracene & 0.760 & & & & 9.13 & & & + & + & + \\
\hline 51 & $\begin{array}{l}\text { Dimethylfluorenes } \\
\text { Trimethylacenaphthene }\end{array}$ & 0.769 & $\begin{array}{r}1.11 \\
M\end{array}$ & $\begin{array}{r}0.43 \\
M\end{array}$ & & & $<0.01$ & - & & $\stackrel{+}{+}$ & $\begin{array}{l}+ \\
+\end{array}$ \\
\hline
\end{tabular}


Table 1. Composition of gel filtration fractions 40 to 45 (contd.).

\begin{tabular}{|c|c|c|c|c|c|c|c|c|c|c|c|}
\hline \multirow{2}{*}{$\begin{array}{c}\text { Peak } \\
\text { No. }\end{array}$} & \multirow{2}{*}{ Compound } & \multirow{2}{*}{$\begin{array}{c}\text { Relative } \\
\text { reten- } \\
\text { tion } \\
\text { timea }\end{array}$} & \multicolumn{6}{|c|}{ Gel fraction } & \multicolumn{3}{|c|}{$\begin{array}{l}\text { Criteria of } \\
\text { identification }\end{array}$} \\
\hline & & & \multicolumn{6}{|c|}{ Percent composition 3} & GC-RTC & UVd & MSe \\
\hline 52 & $\begin{array}{l}\text { Dimethylfiuorene } \\
\text { Trimethylacenaphthene } \\
\text { Dimethyl-unknown of RRT } 0.545\end{array}$ & 0.780 & $\begin{array}{r}1.04 \\
M\end{array}$ & $\begin{array}{r}0.48 \\
M\end{array}$ & 0.01 & 0.03 & 0.03 & $<0.01$ & & $\begin{array}{l}+ \\
+ \\
+\end{array}$ & $\begin{array}{l}+ \\
+ \\
+\end{array}$ \\
\hline 53 & $\begin{array}{l}\text { Dimethylfluorene } \\
\text { Trimethylacenaphthylene }\end{array}$ & 0.794 & $\begin{array}{c}0.90^{h} \\
M\end{array}$ & $\begin{array}{c}0.54 h \\
M\end{array}$ & 0.10 & 0.089 & $<0.01$ & 0.14 & & $\begin{array}{l}+ \\
+\end{array}$ & $\begin{array}{l}+ \\
+\end{array}$ \\
\hline 54 & $\begin{array}{l}\text { Trimethylacenaphthylene } \\
\text { Dimethyifluorene }\end{array}$ & 0.804 & $0.26 h$ & 0.62 & 0.17 & 0.17 & 0.12 & 0.14 & & + & $\stackrel{+}{+}$ \\
\hline 55 & $\begin{array}{l}\text { Dimethylfluorene } \\
\text { Trimethylacenaphthylene }\end{array}$ & 0.810 & $0.66 \mathrm{~h}$ & h & & $g$ & & & & + & $\begin{array}{l}+ \\
+\end{array}$ \\
\hline 56 & $\begin{array}{l}\text { Dimethylbenz(f)indene } \\
\text { Trimethylacenaphthylene }\end{array}$ & 0.821 & $0.58 \mathrm{~h}$ & $0.35^{h}$ & 0.06 & 0.019 & $<0.01$ & - & & $\stackrel{+}{+}$ & $\dot{t}$ \\
\hline 57 & $\begin{array}{l}\text { 2-Methylphenanthrene } \\
\text { 3-Methylphenanthrene } \\
\text { Dimethylfluorene } \\
\text { Trimethylacenaphthylene }\end{array}$ & 0.843 & $\begin{array}{r}3.70 \\
M \\
M\end{array}$ & $\begin{array}{r}5.45 \\
M \\
M\end{array}$ & 5.98 & $\begin{array}{r}3.67 \\
M \\
M\end{array}$ & 1.05 & $\begin{array}{l}0.26 \\
M \\
M\end{array}$ & $\begin{array}{l}+ \\
+\end{array}$ & $\begin{array}{l}+ \\
+ \\
+ \\
+\end{array}$ & $\begin{array}{l}+ \\
+ \\
+ \\
+\end{array}$ \\
\hline 58 & 2-Methylanthracene & 0.849 & 1.49 & 2.45 & 6.15 & 2.66 & 3.14 & 1.09 & + & + & + \\
\hline 59 & $\begin{array}{l}\text { 9-Methylphenanthrene } \\
\text { 1-Methylphenanthrene } \\
\text { 1-Methylanthracene }\end{array}$ & 0.859 & & & & 3.29 & & & + & $\begin{array}{l}+(18) \\
+ \\
+(18)\end{array}$ & $\begin{array}{l}+ \\
+ \\
+\end{array}$ \\
\hline 60 & $\begin{array}{l}\text { 9-Methylanthracene } \\
\text { 4-Methylphenanthrene }\end{array}$ & 0.877 & $\begin{array}{r}0.66 \\
M\end{array}$ & $\begin{array}{r}0.59 \\
M\end{array}$ & 0.12 & $\begin{array}{r}0.23 \\
M\end{array}$ & 0.06 & $<0.01$ & + & $\begin{array}{l}+ \\
+(18)\end{array}$ & $\begin{array}{l}+ \\
+\end{array}$ \\
\hline 61 & $\begin{array}{l}\text { Dimethylphenanthrene } \\
\text { Tetramethylacenaphthene } \\
\text { Tetramethylacenaphthylene }\end{array}$ & 0.881 & $<0.01$ & h & 0.03 & h & $<0.01$ & - & 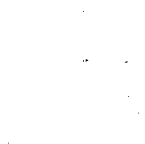 & $\begin{array}{l}+ \\
+ \\
+\end{array}$ & $\begin{array}{l}+ \\
+ \\
+\end{array}$ \\
\hline 62 & $\begin{array}{l}\text { Dimethylphenanthrene } \\
\text { Tetramethylacenaphthene } \\
\text { Tetramethylacenaphthylene }\end{array}$ & 0.897 & $\begin{array}{c}0.03^{h} \\
M \\
M\end{array}$ & $0.36 \mathrm{~h}$ & 0.05 & $\begin{array}{r}0.06 \\
M\end{array}$ & 0.11 & 0.44 & & $\begin{array}{l}+ \\
+ \\
+\end{array}$ & $\begin{array}{l}+ \\
+ \\
+\end{array}$ \\
\hline 63 & $\begin{array}{l}\text { Dimethylphenanthrene. } \\
\text { Dimethylanthracene }\end{array}$ & 0.905 & $<0.01$ & & $<0.01$ & $\begin{array}{r}0.02 \\
M\end{array}$ & $<0.01$ & - & & + & + \\
\hline 64 & $\begin{array}{l}\text { Dimethylphenanthrenes } \\
\text { Dimethylanthracene } \\
\text { Tetramethylacenaphthylene }\end{array}$ & 0.924 & $1.87 \mathrm{~h}$ & $\begin{array}{r}1.24 \\
M\end{array}$ & 0.10 & 0.03 & $<0.01$ & - & & $\begin{array}{l}+ \\
+ \\
+\end{array}$ & $\begin{array}{l}+ \\
+ \\
+\end{array}$ \\
\hline 65 & $\begin{array}{l}\text { Dimethylphenanthrenes } \\
\text { Dimethylanthracene }\end{array}$ & 0.941 & 3.13 & 3.43 & 2.22 & 0.70 & 0.34 & 0.21 & & $\stackrel{+}{+}$ & $\begin{array}{l}+ \\
+\end{array}$ \\
\hline 66 & $\begin{array}{l}\text { Dimethylphenanthrene } \\
\text { Dimethylenephenanthrene }\end{array}$ & 0.951 & $\begin{array}{r}0.62 \\
M\end{array}$ & & & 0.62 & 0.35 & $<0.01$ & & + & $\begin{array}{l}+ \\
+\end{array}$ \\
\hline
\end{tabular}


Table 1 (contd.)

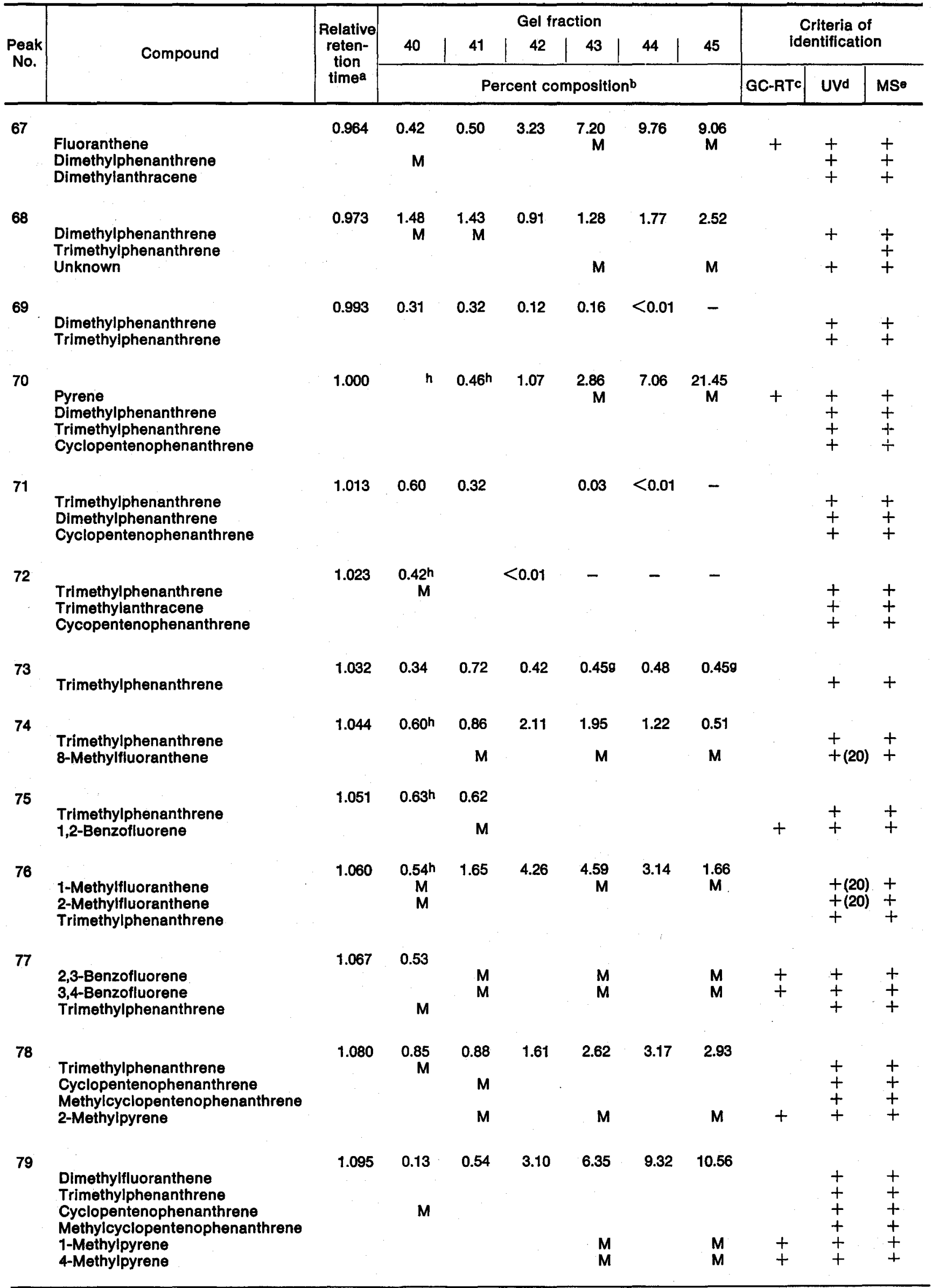


Table 1. Composition of gel filtration fractions 40 to 45 (contd.).

\begin{tabular}{|c|c|c|c|c|c|c|c|c|c|c|c|}
\hline \multirow{2}{*}{$\begin{array}{c}\text { Peak } \\
\text { No. }\end{array}$} & \multirow{2}{*}{ Compound } & \multirow{2}{*}{$\left|\begin{array}{c}\text { Relative } \\
\text { reten- } \\
\text { tion } \\
\text { timea }\end{array}\right|$} & \multicolumn{6}{|c|}{ Gel fraction } & \multicolumn{3}{|c|}{$\begin{array}{l}\text { Criteria of } \\
\text { identification }\end{array}$} \\
\hline & & & \multicolumn{6}{|c|}{ Percent compositionb } & GC-RTC & UVd & MSe \\
\hline 80 & $\begin{array}{l}\text { Dimethylfluoranthene } \\
\text { Cyclopentenophenanthrene } \\
\text { Methylcyclopentenophenanthrene }\end{array}$ & 1.107 & 0.20 & 0.20 & $<0.01$ & - & - & - & & $\begin{array}{l}+ \\
+ \\
+\end{array}$ & $\begin{array}{l}+ \\
+ \\
+\end{array}$ \\
\hline 81 & $\begin{array}{l}\text { Dimethylfluoranthene } \\
\text { Methyl-1,2-benzofluorene } \\
\text { Methylcyclopentenophenanthrene }\end{array}$ & 1.124 & 0.70 & $\begin{array}{r}0.76 \\
M\end{array}$ & 0.35 & 0.08 & 0.14 & $<0.01$ & & $\begin{array}{l}+ \\
+ \\
+\end{array}$ & $\begin{array}{l}+ \\
+ \\
+\end{array}$ \\
\hline 82 & $\begin{array}{l}\text { Dimethylfluoranthenes } \\
\text { Methyl-1,2-benzofluorene } \\
\text { Methyl-2,3-benzofluorene } \\
\text { Methyl-3,4-benzofluorene }\end{array}$ & 1.133 & 1.18 & 1.14 & 0.66 & 0.13 & 0.40 & 0.35 & & $\begin{array}{l}+ \\
+ \\
+ \\
+\end{array}$ & $\begin{array}{l}+ \\
+ \\
+ \\
+\end{array}$ \\
\hline 83 & $\begin{array}{l}\text { Dimethylfluoranthene } \\
\text { Dimethylpyrene } \\
\text { Methyl-1,2-benzofluorene } \\
\text { Methyl-3,4-benzofluorene }\end{array}$ & 1.150 & $\stackrel{0.42^{h}}{M}$ & $\begin{array}{c}0.54 \mathrm{~h} \\
\mathrm{M}\end{array}$ & 0.59 & $\begin{array}{r}0.27 \\
M\end{array}$ & 0.59 & 0.35 & & $\begin{array}{l}+ \\
+ \\
+ \\
+\end{array}$ & $\begin{array}{l}+ \\
+ \\
+ \\
+\end{array}$ \\
\hline 84 & $\begin{array}{l}\text { Dimethylpyrene } \\
\text { Dimethylfluoranthenes } \\
\text { Methylbenzofluorene }\end{array}$ & 1.160 & $\begin{array}{r}0.53 \mathrm{~h} \\
\mathrm{M}\end{array}$ & $\begin{array}{r}0.69 \\
M\end{array}$ & 0.77 & $\begin{array}{r}0.62 \\
M\end{array}$ & 0.85 & 0.12 & & $\begin{array}{l}+ \\
+ \\
+\end{array}$ & $\begin{array}{l}+ \\
+ \\
+\end{array}$ \\
\hline 85 & $\begin{array}{l}\text { Dimethylpyrenes } \\
\text { Dimethylfluoranthene } \\
\text { Methylbenzofluorene } \\
\text { Benzo }(g, h, l) \text { fluoranthene }\end{array}$ & 1.167 & $\begin{array}{c}0.44 h \\
M\end{array}$ & $\begin{array}{c}0.67 \mathrm{~h} \\
\mathrm{M}\end{array}$ & 1.06 & $\begin{array}{r}0.96 \\
M\end{array}$ & 0.97 & $\begin{array}{c}2.55 \\
M\end{array}$ & + & $\begin{array}{l}+ \\
+ \\
+ \\
+\end{array}$ & $\begin{array}{l}+ \\
+ \\
+ \\
+\end{array}$ \\
\hline 86 & $\begin{array}{l}\text { Dimethylpyrenes } \\
\text { Methylbenzofluorene } \\
\text { Trimethylfluoranthene }\end{array}$ & 1.186 & $\begin{array}{c}0.32^{h} \\
M\end{array}$ & $\begin{array}{r}0.56 \\
M\end{array}$ & 1.48 & $\begin{array}{r}1.87 \\
M\end{array}$ & .1 .63 & $\begin{array}{c}0.85 \\
M\end{array}$ & & $\begin{array}{l}+ \\
+ \\
+\end{array}$ & $\begin{array}{l}+ \\
+ \\
+\end{array}$ \\
\hline 87 & Methylbenzofluorene & 1.207 & 0.58 & 0.87 & 2.35 & 3.85 & 5.76 & 5.62 & & + & + \\
\hline 88 & 1,2-Benzanthracene & & & $\mathbf{M}$ & & M & & & + & + & + \\
\hline 89 & Chrysene & & & $\mathbf{M}$ & - & $\mathbf{M}$ & & M & + & + & + \\
\hline 90 & $\begin{array}{l}\text { Triphenylene } \\
\text { Trimethylfluoranthene } \\
\text { Trimethylpyrene }\end{array}$ & & $\mathbf{M}$ & & & $\mathbf{M}$ & & $\mathbf{M}$ & + & $\begin{array}{l}+ \\
+ \\
+\end{array}$ & $\begin{array}{l}+ \\
+ \\
+\end{array}$ \\
\hline 91 & $\begin{array}{l}\text { Trimethylpyrene } \\
\text { Trimethylftuoranthene } \\
\text { Dimethylbenzofluorene } \\
\text { 3,4-Dimethylenepyrene }\end{array}$ & 1.230 & $\begin{array}{r}0.18 \\
M\end{array}$ & $<0.01$ & 0.12 & 0.02 & 0.87 & $\begin{array}{c}1.32 \\
M\end{array}$ & & $\begin{array}{l}+ \\
+ \\
+ \\
+\end{array}$ & $\begin{array}{l}+ \\
+ \\
+ \\
+\end{array}$ \\
\hline 92 & $\begin{array}{l}\text { Trimethylpyrene } \\
\text { Trimethylfluoranthene }\end{array}$ & 1.236 & 0.10 & 0.39 & & & $<0.01$ & - & & $\begin{array}{l}+ \\
+\end{array}$ & + \\
\hline 93 & Trimethylpyrenes & 1.246 & 0.13 & 0.35 & 0.33 & 0.02 & 0.33 & 0.40 & & + & + \\
\hline
\end{tabular}


Table 1 (contd.)

\begin{tabular}{|c|c|c|c|c|c|c|c|c|c|c|c|}
\hline \multirow{2}{*}{$\begin{array}{c}\text { Peak } \\
\text { No. }\end{array}$} & \multirow{2}{*}{ Compound } & \multirow{2}{*}{$\begin{array}{c}\text { Relative } \\
\text { reten- } \\
\text { tion } \\
\text { timea }\end{array}$} & \multicolumn{6}{|c|}{ Gel fraction } & \multicolumn{3}{|c|}{$\begin{array}{l}\text { Criteria of } \\
\text { identification }\end{array}$} \\
\hline & & & \multicolumn{6}{|c|}{ Percent composition $b$} & GC-RTC & UVd & MSe \\
\hline 94 & $\begin{array}{l}\text { Trlmethylpyrenes } \\
\text { Methyl-1,2-benzanthracene } \\
\text { Methylbenzo(g,h,i)fluoranthene }\end{array}$ & 1.255 & $\begin{array}{r}0.10 \\
M\end{array}$ & $\begin{array}{r}0.18 \\
M\end{array}$ & & $\begin{array}{r}0.16 \\
M\end{array}$ & 0.58 & 0.64 & & $\begin{array}{l}+ \\
+ \\
+\end{array}$ & $\begin{array}{l}+ \\
+ \\
+\end{array}$ \\
\hline 95 & $\begin{array}{l}\text { 2-Methylchrysene } \\
\text { 3-Methylchrysene } \\
\text { Methyl-1,2-benzanthracene } \\
\text { Methyltriphenylene } \\
\text { Methylbenzo(g,h,i)fluoranthene } \\
\text { Trimethylpyrene }\end{array}$ & 1.276 & $\begin{array}{r}0.46 \\
M\end{array}$ & $\begin{array}{r}0.77 \\
M\end{array}$ & 1.28 & $\begin{array}{r}0.84 \\
M\end{array}$ & 0.94 & 0.40 & $\begin{array}{l}+ \\
+\end{array}$ & $\begin{array}{l}+ \\
+ \\
+ \\
+ \\
+ \\
+\end{array}$ & $\begin{array}{l}+ \\
+ \\
+ \\
+ \\
+ \\
+\end{array}$ \\
\hline 96 & $\begin{array}{l}\text { 4-Methylchrysene } \\
\text { Trimethylpyrene } \\
\text { Tetramethylpyrene } \\
\text { Methyl-1,2-benzanthracenes }\end{array}$ & 1.288 & $\begin{array}{r}0.10 \\
M\end{array}$ & $\begin{array}{r}0.18 \\
M\end{array}$ & 0.92 & $\begin{array}{r}0.25 \\
M\end{array}$ & 1.36 & 0.49 & + & $\begin{array}{l}+ \\
+ \\
+ \\
+\end{array}$ & $\begin{array}{l}+ \\
+ \\
+ \\
+\end{array}$ \\
\hline 97 & $\begin{array}{l}\text { 1-Methylchrysene } \\
\text { 6-Methylchrysene } \\
\text { Methyl-1,2-benzanthracene } \\
\text { Trimethylpyrene } \\
\text { Tetramethylpyrene } \\
\text { Methyltriphenylene }\end{array}$ & 1.294 & 0.08 & & & 0.52 & & 0.93 & + & $\begin{array}{l}+ \\
+ \\
+ \\
+ \\
+ \\
+\end{array}$ & $\begin{array}{l}+ \\
+ \\
+ \\
+ \\
+ \\
+\end{array}$ \\
\hline 98 & $\begin{array}{l}\text { Dimethylchrysene } \\
\text { Tetramethylpyrene } \\
\text { Trimethylpyrene } \\
\text { 3,4-Trimethylenepyrene }\end{array}$ & 1.310 & 0.02 & 0.01 & 0.27 & 0.07 & 0.80 & 0.61 & & $\begin{array}{l}+ \\
+ \\
+ \\
+(21)\end{array}$ & $\begin{array}{l}+ \\
+ \\
+ \\
+\end{array}$ \\
\hline 99 & $\begin{array}{l}\text { Dimethylchrysene } \\
\text { Dimethyl-1,2-benzanthracene } \\
\text { Tetramethylpyrene }\end{array}$ & 1.322 & $\begin{array}{r}0.04 \\
M\end{array}$ & & & 0.03 & $<0.01$ & - & & $\begin{array}{l}+ \\
+ \\
+\end{array}$ & $\begin{array}{l}+ \\
+ \\
+\end{array}$ \\
\hline 100 & $\begin{array}{l}\text { Dimethylchrysenes } \\
\text { Dimethyl-1,2-benzanthracenes } \\
\text { Dimethyltriphenylene } \\
\text { Tetramethylpyrene }\end{array}$ & 1.336 & $\begin{array}{r}0.25 \\
M\end{array}$ & $\begin{array}{r}0.17 \\
M\end{array}$ & 0.07 & $\begin{array}{r}0.02 \\
M\end{array}$ & 0.56 & $\begin{array}{c}0.64 \\
M\end{array}$ & & $\begin{array}{l}+ \\
+ \\
+ \\
+\end{array}$ & $\begin{array}{l}+ \\
+ \\
+ \\
+\end{array}$ \\
\hline 101 & $\begin{array}{l}\text { Dimethylchrysenes } \\
\text { Dimethyl-1,2-benzanthracene } \\
\text { Dimethyltriphenylene }\end{array}$ & 1.359 & $\begin{array}{r}0.19 \\
M\end{array}$ & $\begin{array}{r}0.27 \\
M\end{array}$ & 0.14 & 0.02 & 0.41 & 0.20 & & $\begin{array}{l}+ \\
+ \\
+\end{array}$ & $\begin{array}{l}+ \\
+ \\
+\end{array}$ \\
\hline 102 & $\begin{array}{l}\text { Dimethylchrysene } \\
\text { Dimethyl-1,2-benzanthracene } \\
\text { Dimethyltriphenylene } \\
\text { Trimethylchrysene } \\
\text { Trimethyl-1,2-benzanthracene } \\
\text { Trimethyltriphenylene }\end{array}$ & 1.371 & $\begin{array}{r}0.17 \\
M\end{array}$ & $\begin{array}{r}0.08 \\
M\end{array}$ & 0.04 & 0.03 & $<0.01$ & - & . & $\begin{array}{l}+ \\
+ \\
+ \\
+ \\
+ \\
+\end{array}$ & $\begin{array}{l}+ \\
+ \\
+ \\
+ \\
+ \\
+\end{array}$ \\
\hline 103 & $\begin{array}{l}\text { Benzo(b)fluoranthene } \\
\text { Benzo(j)fluoranthene } \\
\text { Benzo(k)fluoranthene } \\
\text { Dimethylchrysene } \\
\text { Trimethylchrysene }\end{array}$ & 1.384 & $\begin{array}{r}0.02 \\
M \\
M\end{array}$ & 0.01 & 0.30 & $\begin{array}{r}0.71 \\
M \\
M \\
M\end{array}$ & 2.24 & $\begin{array}{c}3.31 \\
M \\
M \\
M\end{array}$ & + & $\begin{array}{l}\cdots \\
+ \\
+(21) \\
+(21) \\
+ \\
+\end{array}$ & $\begin{array}{l}+ \\
+ \\
+ \\
+ \\
+\end{array}$ \\
\hline
\end{tabular}


Table 1. Composition of gel filtration tractions 40 to 45 (contd.).

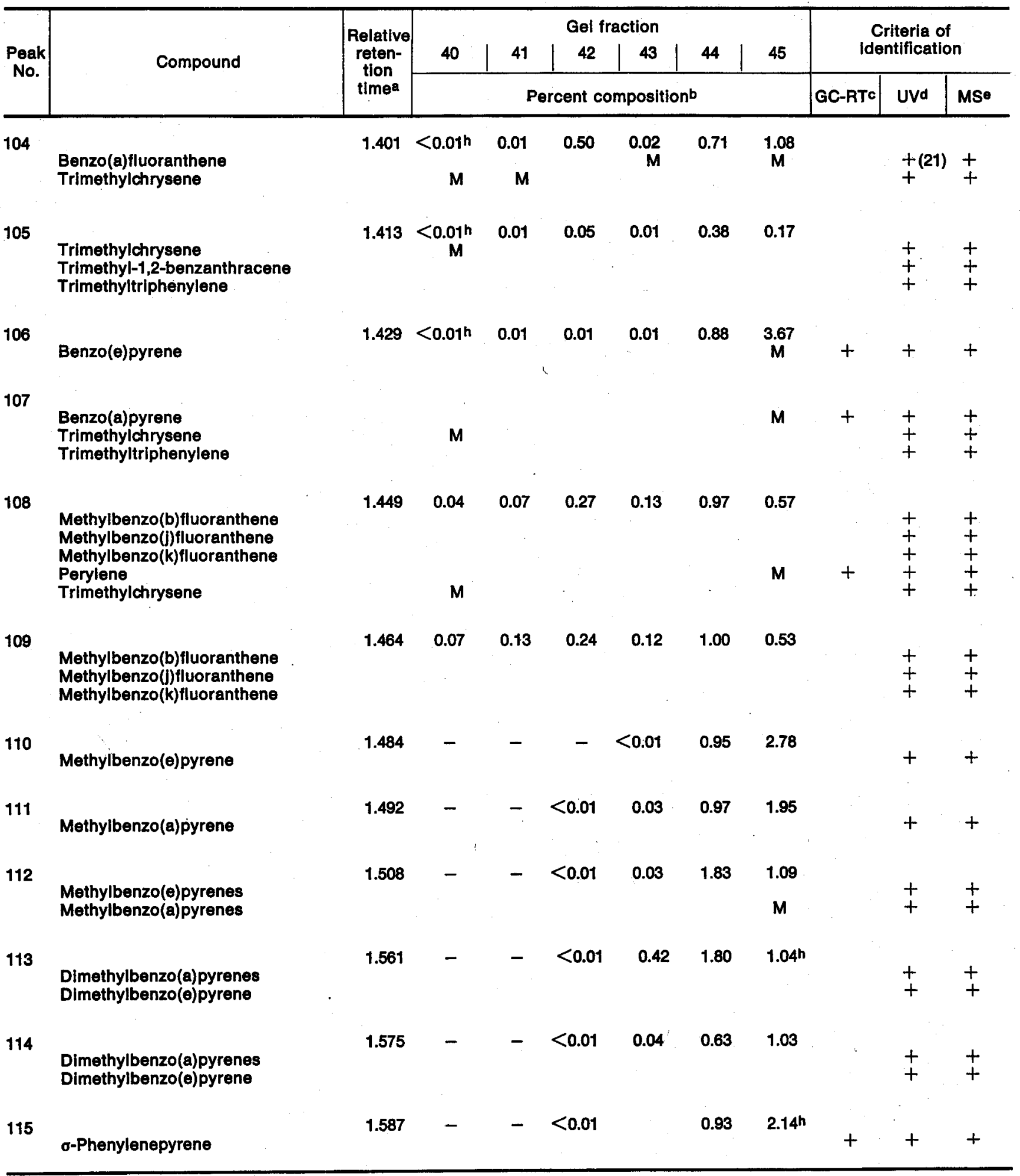

a: Relative to pyrene; a factor of $\mathbf{8 0 . 6}$ converts RRT to minutes from point of injection.

b: Based on total GC volatiles in gel fraction, assuming unitary detector response; represents total percent composition of all components Ilsted tor GC peak(s) up to next listed percentage.

c: QC retention time identical to standard.

d: UV spectra identical to standard, identical to literature, or analogous to parent compound.

o: Molecular ion and fragmentation pattern correlation.

$f$ : Mejor component, greater than $40 \%$ of composition, subsequently denoted by " $M$ ".

g: Major component unidentlfied.

h: Contains other unidentifled material.

M: Major component, greater than $40 \%$ of composition. 
Table 2. PAH identiflcation dataa.

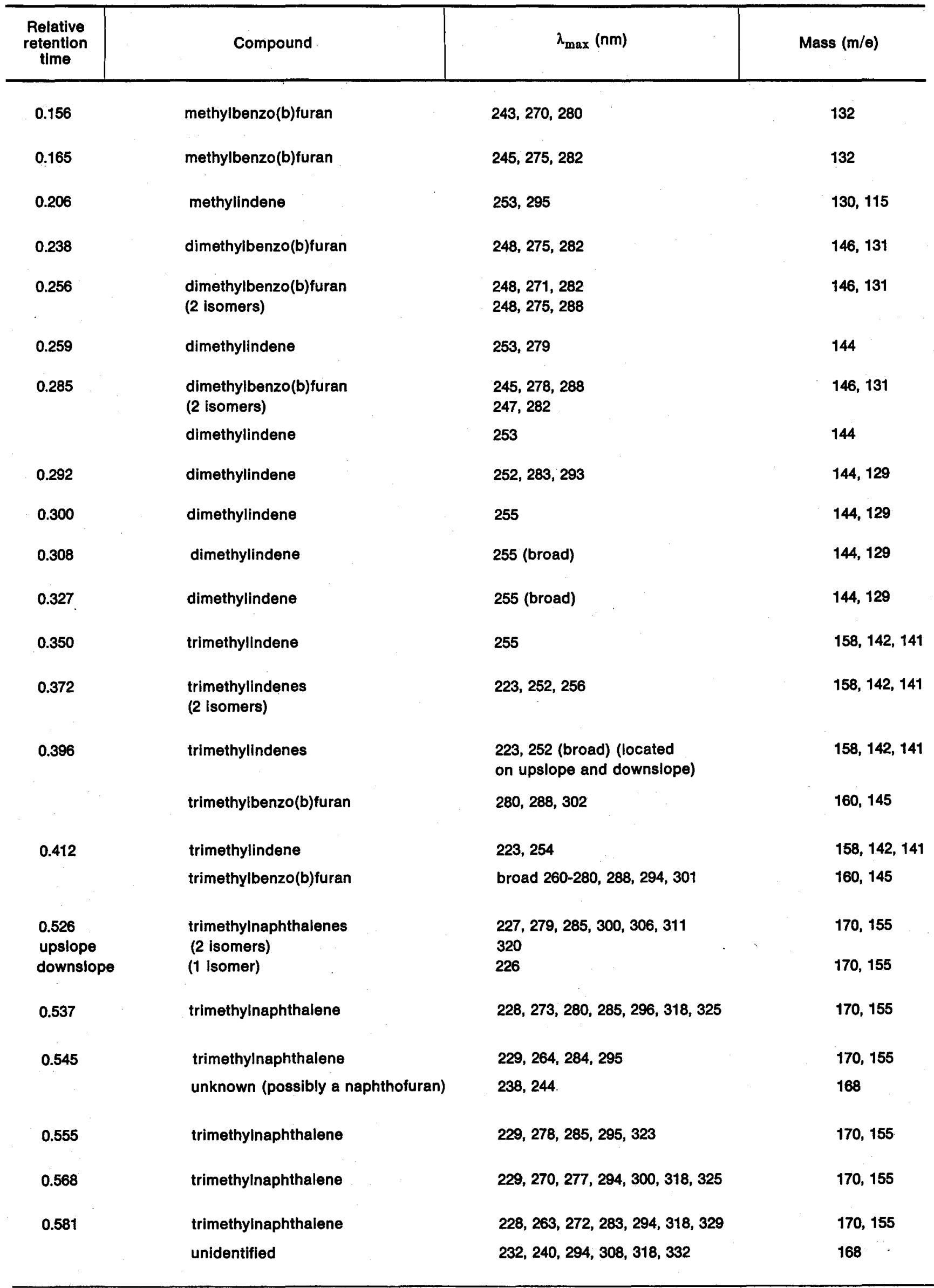


Table 2. PAH identification data (contd.).

\begin{tabular}{|c|c|c|c|}
\hline $\begin{array}{l}\text { Relative } \\
\text { retention } \\
\text { time }\end{array}$ & Compound & $\lambda_{\max }(\mathrm{nm})$ & Mass (m/e) \\
\hline \multirow[t]{2}{*}{0.588} & methylacenaphthylene & $233,315,325,343$ & 166,165 \\
\hline & trimethylnaphthalene & 226,324 & 170,155 \\
\hline \multirow[t]{2}{*}{0.596} & $\begin{array}{l}\text { methylacenaphthylene } \\
\text { ( } 2 \text { isomers) }\end{array}$ & $\begin{array}{l}231,233,300,309,315,323 \\
330,344\end{array}$ & 166 \\
\hline & trimethylnaphthalene & $228,308,314,323$ & 170,155 \\
\hline \multirow[t]{2}{*}{0.612} & methylacenaphthene & $228,280,290,308,317,323$ & $168,167,166$ \\
\hline & unknown (possibly benz(e)indene) & $229,245,254$, broad 280,320 & 166,165 \\
\hline \multirow[t]{2}{*}{0.629} & methyldibenzofuran & $210,219,242,250,286,296,307$ & 182,181 \\
\hline & $\begin{array}{l}\text { unknown (possibly } \\
\text { methylbenz(e)indene) }\end{array}$ & $236,244,254,276,287,300$ & 180 \\
\hline \multirow[t]{2}{*}{0.647} & $\begin{array}{l}\text { methyl-unknown of RRT } 0.545 \\
\text { (possibly a methyinaphthofuran) }\end{array}$ & $230,238,246$, broad $270-285$ & 182,167 \\
\hline & dimethylacenaphthene & $308,314,321,330$ & $182,167,152$ \\
\hline \multirow[t]{2}{*}{0.660} & $\begin{array}{l}\text { methyl-unknown of RRT } 0.545 \\
\text { (possibly a methylnaphthofuran) }\end{array}$ & $240,246,247$ & 182 \\
\hline & dimethylacenaphthene & $231,211,319,326$ & $182,181,180$ \\
\hline 0.671 & dimethylacenaphthene & $232,240,325$ & $182,181,180$ \\
\hline \multirow[t]{2}{*}{0.682} & dimethylacenaphthylene & $235,321,326,336$ & 180 \\
\hline & $\begin{array}{l}\text { methyl-unknown of RRT } 0,545 \\
\text { (possibly a methylnaphthofuran) }\end{array}$ & $210,239,243$ & 182 \\
\hline \multirow[t]{2}{*}{0.694} & dimethylacenaphthylene & 233, broad 322,334 & 180 \\
\hline & methyldibenzofuran & 248,256 & 182 \\
\hline 0.704 & $\begin{array}{l}\text { dimethylacenaphthylenes } \\
\text { ( } 2 \text { isomers) }\end{array}$ & 232,235 , broad 328,345 & 180,165 \\
\hline \multirow{2}{*}{. $\quad \therefore$} & dimethylacenaphthene & 230,326 & 182 \\
\hline & dimethyldibenzofuran & 248,257 & 196 \\
\hline \multirow[t]{2}{*}{0.716} & dimethylacenaphthene & 232 , broad 328 & 182,167 \\
\hline & $\begin{array}{l}\text { methylbenz(f)indene } \\
\text { dimethyldibenzofuran }\end{array}$ & $\begin{array}{l}278,288,300 \\
245,255\end{array}$ & $\begin{array}{l}180,165 \\
196,181\end{array}$ \\
\hline \multirow[t]{4}{*}{0.731} & methylbenz(f) indene & broad 238-245, 277, 287, 300 & 180,165 \\
\hline & dimethyldibenzofuran & 248,258 & 196,181 \\
\hline & dimethylacenaphthene & 233, broad 325 & 182,167 \\
\hline & tetramethyInaphthalene & $231,257,261,275,280,285$ & 184 \\
\hline \multirow{3}{*}{$\begin{array}{r}0.745 \\
2.1\end{array}$} & trimethylacenaphthene & 232,325 & 196,181 \\
\hline & trimethylacenaphthylene & 232,325 & 194 \\
\hline & methylbenz(f) indene & $278,288,300$ & 180,165 \\
\hline$\therefore$ & $\begin{array}{l}\text { dimethyl-unknown of RRT } 0.545 \\
\text { (possibly a dimethylnaphthofuran) }\end{array}$ & 248,257 & 196, 181 \\
\hline
\end{tabular}


Table 2 (contd.)

\begin{tabular}{|c|c|c|c|}
\hline $\begin{array}{c}\text { Relative } \\
\text { retention } \\
\text { time }\end{array}$ & Compound & $\lambda_{\max }(\mathrm{nm})$ & Mass (m/e) \\
\hline 0.754 & trimethylacenaphthene & 234, broad 324 & 196,181 \\
\hline \multirow[t]{2}{*}{0.769} & $\begin{array}{l}\text { dimethylfluorenes } \\
\text { (2 isomers) }\end{array}$ & $266,294,299,306$ & 194,179 \\
\hline & trimethylacenaphthene & 231,328 & 196 \\
\hline \multirow[t]{3}{*}{0.780} & dimethylfluorene & $268,289,301$ & 194,179 \\
\hline & $\begin{array}{l}\text { dimethyl-unknown of RRT } 0.545 \\
\text { (possibly a dimethylnaphthofuran) }\end{array}$ & 246, broad 280 & 196 \\
\hline & trimethylacenaphthene & 236,326 & 196 \\
\hline \multirow[t]{3}{*}{0.794} & dimethylfluorene & $209,258,264,276,306$ & 194,179 \\
\hline & trimethylacenaphthylene & $234,325,345$ & 194 \\
\hline & unidentified & 258,292 & 196,181 \\
\hline \multirow[t]{3}{*}{0.804} & trimethylacenaphthylene & $232,300,312,328$ & 194,179 \\
\hline & dimethylfluorene & 204,305 & 194,179 \\
\hline & unidentifled & & 196 \\
\hline \multirow[t]{3}{*}{0.810} & dimethylfluorene & $208,261,300$ & 194,179 \\
\hline & trimethylacenaphthylene & 232,325 & 194 \\
\hline & unidentified & $237,251,262,284,292,300,311$ & 198, 196,192 \\
\hline \multirow[t]{3}{*}{0.821} & trimethylacenaphthylene & 234 , broad $320-340$ & 194,179 \\
\hline & dimethylbenz(f)indene & $279,288,300$ & 196,181 \\
\hline & unidentified & $236,251,289$ & 208,198 \\
\hline $\begin{array}{l}0.843 \text { (front } \\
\text { shoulder) }\end{array}$ & dimethylfluorene & $203,259,289,292,302$ & 194, 179 \\
\hline (front shoulder) & trimethylacenaphthylene & 235,330 & 194,179 \\
\hline \multirow[t]{3}{*}{0.881} & dimethyiphenanthrene & $252,275,283,295$ & 206, 191 \\
\hline & tetramethylacenaphthene & 238 , broad $310-340$ & 208 \\
\hline & unidentified & 246 & 204 \\
\hline \multirow[t]{4}{*}{0.897} & dimethylphenanthrene & $252,304,310,318$ & 206 \\
\hline & tetramethylacenaphthene & 232 & 210 \\
\hline & tetramethylacenaphthylene & 238 & 208 \\
\hline & unidentified & 275,288 & 212,192 \\
\hline \multirow[t]{2}{*}{0.905} & dimethylphenanthrene & 275,288 & 212,192 \\
\hline & dimethylanthracene & & \\
\hline \multirow[t]{3}{*}{0.924} & $\begin{array}{l}\text { dimethylphenanthrenes } \\
\text { ( } 3 \text { isomers) }\end{array}$ & $\begin{array}{l}252,275,283,293 \\
253,277,284,296 \\
252,275,285,296\end{array}$ & 206, 191 \\
\hline & dimethylanthracene & $255,262,370,381$ & 206,191 \\
\hline & tetramethylacenaphthylene & 242,328 & 208,193 \\
\hline
\end{tabular}


Table 2. PAH identification data (contd.).

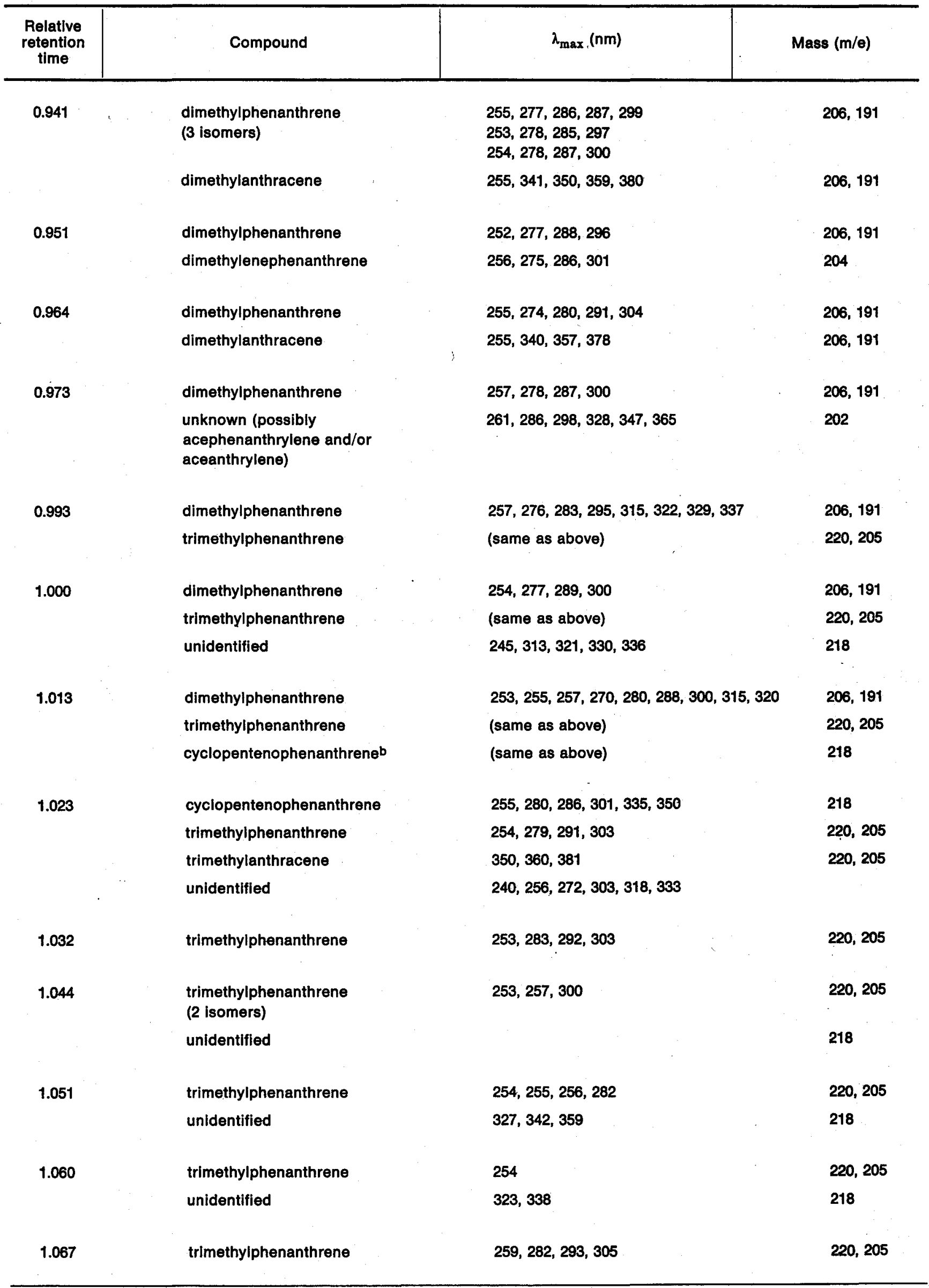


Table 2 (contd.)

\begin{tabular}{|c|c|c|c|}
\hline $\begin{array}{l}\text { Relative } \\
\text { retention } \\
\text { time }\end{array}$ & Compound & $\lambda_{\max }(n m)$ & Mass (m/e) \\
\hline \multirow[t]{3}{*}{1.080} & trimethylphenanthrene & $258,278,288,300,335,351$ & 220,205 \\
\hline & cyclopentenophenanthrene & (same as above) & 218 \\
\hline & methylcyclopentenophenanthrene & (same as above) & 232 \\
\hline \multirow[t]{4}{*}{1.095} & dimethylfluoranthene & $238,275,286$ & 230 \\
\hline & cyclopentenophenanthrene & $253,258,261,300$ & 218 \\
\hline & methylcyciopentenophenanthrene & (same as above) & 234 \\
\hline & trimethylphenanthrene & (same as above) & 220,205 \\
\hline \multirow[t]{3}{*}{1.107} & dimethylfluoranthene & $236,277,282,288,323,342,359$ & 230,215 \\
\hline & methylcyclopentenophenanthrene & 254,300 & 232,217 \\
\hline & cyclopentenophenanthrene & (same as above) & 218 \\
\hline \multirow[t]{3}{*}{1.124} & dimethylfluoranthene & $237,279,284,290,342,36$ & 230,215 \\
\hline & methyl-1,2-benzofluorene & 262 & 230,215 \\
\hline & methylcyciopentenophenanthrene & 254,300 & 232,217 \\
\hline 1.133 & $\begin{array}{l}\text { dimethylfluoranthene } \\
(2 \text { isomers) }\end{array}$ & $\begin{array}{l}238,276,288,364 \\
240,280,291,362\end{array}$ & 230,215 \\
\hline \multirow[t]{5}{*}{1.150} & dimethylpyrene & $247,277,310,323,337$ & 230,215 \\
\hline & dimethylfiuoranthene & $240,272,290,364$ & 230,215 \\
\hline & methyl-1,2-benzofluorene & $254,260,263$ & 230,215 \\
\hline & methyl-3,4-benzofluorene & $311,322,337$ & 230,215 \\
\hline & unidentified & 301 & 246,232 \\
\hline \multirow[t]{4}{*}{1.160} & dimethylpyrene & $245,266,277,307,323,339$ & 230,215 \\
\hline & $\begin{array}{l}\text { dimethylfluoranthene } \\
\text { ( } 2 \text { isomers) }\end{array}$ & $\begin{array}{l}242,282,361 \\
247,290\end{array}$ & 230,215 \\
\hline & methylbenzofluorene & 254,262 & 230,215 \\
\hline & unidentified & & 246,232 \\
\hline \multirow[t]{5}{*}{1.167} & $\begin{array}{l}\text { dimethylpyrene } \\
\text { ( } 2 \text { isomers) }\end{array}$ & $\begin{array}{l}243,266,277,307,309,323 \\
324,338,342\end{array}$ & 230,215 \\
\hline & dimethylfluoranthene & 288 & 230,215 \\
\hline & methylbenzofluorenes & 254,262 & 230,215 \\
\hline & $\begin{array}{l}\text { unknown (possibly a } \\
\text { methylcyclopentenophenanthrene) }\end{array}$ & & 232,217 \\
\hline & $\begin{array}{l}\text { unknown (possibly a dimethyl- } \\
\text { cyclopentenophenanthrene) }\end{array}$ & & 246 \\
\hline \multirow[t]{4}{*}{1.186} & $\begin{array}{l}\text { dimethylpyrene } \\
\text { ( } 2 \text { isomers) }\end{array}$ & $\begin{array}{l}243,266,277,323,327,338 \\
343\end{array}$ & 230,215 \\
\hline & methylbenzofluorene & 255,263 & 230,215 \\
\hline & trimethylfluoranthene & $245,288,292$ & 244 \\
\hline & $\begin{array}{l}\text { unknown (possibly a } \\
\text { methylcyclopentenophenanthrene) }\end{array}$ & & 232,217 \\
\hline
\end{tabular}


Table 2. PAH identification dataa (contd.).

\begin{tabular}{|c|c|c|c|}
\hline $\begin{array}{l}\text { Relative } \\
\text { retention } \\
\text { time }\end{array}$ & Compound & $\lambda_{\max }(\mathrm{nm})$ & Mass $(\mathrm{m} / \mathrm{e})$ \\
\hline \multirow[t]{3}{*}{1.207} & methylbenzofluorene & 255,265 & 230,215 \\
\hline & trimethylfluoranthene & 290,363 & 244 \\
\hline & $\begin{array}{l}\text { trimethylpyrene } \\
\text { ( } 2 \text { isomers) }\end{array}$ & $245,276,326,338,341$ & 244,229 \\
\hline \multirow[t]{4}{*}{1.230} & trimethylpyrene & $243,277,312,324,339$ & 244 \\
\hline & trimethylfluoranthene & 290,293 & 244,299 \\
\hline & dimethylbenzofluorene & 254,265 & 244,229 \\
\hline & 3,4-dimethylenepyrene & $242,254,265,275,313,327,343$ & 228 \\
\hline \multirow[t]{2}{*}{1.236} & trimethylpyrene & $243,266,277,313,324,339$ & 244,229 \\
\hline & trimethylfluoranthene & 246,289 & 244 \\
\hline 1.246 & $\begin{array}{l}\text { trimethylpyrenes } \\
\text { (2 isomers) }\end{array}$ & $\begin{array}{l}245,255,265,279,324,340 \\
245,257,267,280,326,345\end{array}$ & 244,229 \\
\hline \multirow[t]{3}{*}{1.255} & $\begin{array}{l}\text { trimethylpyrene } \\
\text { ( } 2 \text { isomers) }\end{array}$ & $\begin{array}{l}245,257,267,279,313,328 \\
340,344\end{array}$ & 244,229 \\
\hline & methyl-1,2-benzanthracene & 290 & 242 \\
\hline & methylbenzo $(g, h, i)$ fluoranthene & 234,290 & 240 \\
\hline \multirow[t]{4}{*}{1.276} & trimethylpyrene & $244,278,329,346$ & 244,229 \\
\hline & methyl-1,2-benzanthracerie & 277,288 & 242 \\
\hline & methylbenzo(g, h, l)fluoranthene & 233 & 240 \\
\hline & methyltriphenylene & 249,259 & 242 \\
\hline \multirow[t]{3}{*}{1.288} & trimethylpyrene & $245,326,345$ & 244,229 \\
\hline & tetramethylpyrene & (same as above) & 258,243 \\
\hline & $\begin{array}{l}\text { methyl-1,2-benzanthracene } \\
\text { (2 isomers) }\end{array}$ & $270,289,293$ & 242 \\
\hline \multirow[t]{3}{*}{1.294} & trimethylpyrene & $245,279,330,345$ & 244,229 \\
\hline & tetramethylpyrene & (same as above) & 258,243 \\
\hline & methyl-1,2-benzanthracene & 279,290 & 242,227 \\
\hline & methyltriphenylene & 249,259 & 242 \\
\hline \multirow[t]{3}{*}{1.310} & trimethylpyrene & $244,279,327,343$ & 244,229 \\
\hline & tetramethylpyrene & (same as above) & 258,243 \\
\hline & dimethylchrysene & 257,268 & 256,239 \\
\hline \multirow[t]{3}{*}{1.322} & dimethylchrysene & $259,269,320$ & 256,241 \\
\hline & dimethyl-1,2-benzanthracene & 279,289 & 256,241 \\
\hline & tetramethylpyrene & $279,330,344$ & 258,243 \\
\hline
\end{tabular}


Table 2 (contd.)

\begin{tabular}{|c|c|c|c|}
\hline $\begin{array}{l}\text { Relative } \\
\text { retention } \\
\text { time }\end{array}$ & Compound & $\lambda_{\max }(n m)$ & $s(\mathrm{~m} / \theta)$ \\
\hline \multirow[t]{4}{*}{1.366} & $\begin{array}{l}\text { dimethylchrysene } \\
\text { ( } 2 \text { isomers by GC) }\end{array}$ & $259,269,319$ & 256,241 \\
\hline & $\begin{array}{l}\text { dimethyl-1,2-benzanthracene } \\
\text { ( } 2 \text { isomers by } G C)\end{array}$ & 279,289 & 256,241 \\
\hline & dimethyltriphenylene & 249,260 & 256,241 \\
\hline & tetramethylpyrene & $249,279,330,344$ & 258,243 \\
\hline \multirow[t]{3}{*}{1.359} & $\begin{array}{l}\text { dimethylchrysene } \\
\text { ( } 2 \text { isomers by GC) }\end{array}$ & $250,260,310,324$ & 256,241 \\
\hline & dimethyl-1,2-benzanthracene & 270,280 & 256,241 \\
\hline & dimethyltriphenylene & 249,259 & 256,241 \\
\hline \multirow[t]{4}{*}{1.371} & dimethylchrysene & $259,270,313,328$ & 256 \\
\hline & trimethylchrysene & (same as above) & 270 \\
\hline & $\begin{array}{l}\text { di- and/or trimethyl- } \\
\text { 1,2-benzanthracene }\end{array}$ & 280,288 & 256,270 \\
\hline & di- and/or trimethyltriphenylene & 248,259 & 256,270 \\
\hline \multirow[t]{2}{*}{1.384} & dimethylchrysene & $261,270,325$ & 256 \\
\hline & trimethylchrysene & (same as above) & 270 \\
\hline \multirow[t]{2}{*}{1.401} & trimethylchrysene & 261,270 & 270 \\
\hline & unidentified & & 268 \\
\hline \multirow[t]{4}{*}{1.413} & trimethylchrysene & 261,271 & 270 \\
\hline & trimethyl-1,2-benzanthracene & 280,292 & 270 \\
\hline & trimethyltriphenylene & 261 & 270 \\
\hline & unidentified & & 268 \\
\hline \multirow[t]{3}{*}{1.429} & trimethylchrysene & 261,272 & 270 \\
\hline & trimethyltriphenylene & 252,261 & 270 \\
\hline & unidentified & 224 & 268 \\
\hline \multirow[t]{5}{*}{1.449} & trimethylchrysene & 262,272 & 270 \\
\hline & methylbenzo(b)fluoranthene & $254,276,292,302$ & 266 \\
\hline & methylbenzo(j)fluoranthene & $223,242,319,332,345,364,382$ & 266 \\
\hline & methylbenzo(k)fluoranthene & 240,402 & 266 \\
\hline & methylbenzo(a)fluoranthene & 256,363 & 266 \\
\hline \multirow[t]{3}{*}{1.464} & methylbenzo(b)fluoranthene & $255,290,301$ & 266 \\
\hline & methylbenzo(j)fluoranthene & $242,308,317,332,384$ & 266 \\
\hline & methylbenzo(k)fluoranthene & $240,308,402$ & 266 \\
\hline 1.489 & methylbenzo(e)pyrene & $204,215,223,237,257,266,278,289,317,331$ & 266 \\
\hline 1.492 & methylbenzo(a)pyrene & $255,267,285,297,348,366,386$ & 266 \\
\hline
\end{tabular}


Table 2. PAH identification dataa (contd.).

\begin{tabular}{|c|c|c|c|}
\hline $\begin{array}{c}\text { Relative } \\
\text { retention } \\
\text { time }\end{array}$ & Compound & $\lambda_{\max }(n m)$ & Mass $(\mathrm{m} / \mathrm{e})$ \\
\hline \multirow[t]{2}{*}{1.508} & $\begin{array}{l}\text { methylbenzo(a)pyrene } \\
\text { ( } 2 \text { isomers) }\end{array}$ & $\begin{array}{l}254,265,285,296,348,366 \\
\text { broad } 380-387\end{array}$ & 266 \\
\hline & $\begin{array}{l}\text { methylbenzo(e)pyrene } \\
\text { ( } 2 \text { isomers) }\end{array}$ & $\begin{array}{l}204,279,290,318,331 \\
280,290,320,336\end{array}$ & 266 \\
\hline \multirow[t]{2}{*}{1.561} & $\begin{array}{l}\text { dimethylbenzo(a)pyrene } \\
\text { ( } 2 \text { isomers) }\end{array}$ & $\begin{array}{l}257,266,367,386 \\
257,267,368,388\end{array}$ & 280,265 \\
\hline & dimethylbenzo(e)pyrene & $204,224,280,292,319,333$ & 280,265 \\
\hline \multirow[t]{3}{*}{1.575} & $\begin{array}{l}\text { dimethylbenzo(a)pyrene } \\
(2 \text { isomers) }\end{array}$ & $296,365,367,389$ & 280,265 \\
\hline & dimethylbenzo(e)pyrene & $204,224,281,291,332,337$ & 280,265 \\
\hline & unidentified & 257 & 276 \\
\hline
\end{tabular}

a: This table presents only data for compounds whose GC retention time and/or literature UV data are lacking. $\mathrm{b}$ : One of several possible isomeric phenanthrenes with $\mathrm{m} / \mathrm{e} 218$. 